\title{
WENO Interpolation-Based and Upwind-Biased Free-Stream Preserving Nonlinear Schemes
}

\author{
Qin $\mathrm{Li}^{1,2,3, *}$, Dong Sun ${ }^{1}$ and Fengyuan $\mathrm{Xu}^{3}$ \\ ${ }^{1}$ State Key Laboratory of Aerodynamics, China Aerodynamics Research and \\ Development Center, Mianyang, Sichuan 621000, China \\ ${ }^{2}$ Key Laboratory of Aerodynamics Noise Control, China Aerodynamics Research \\ and Development Center, Mianyang, Sichuan 621000, China \\ ${ }^{3}$ School of Aerospace Engineering, Xiamen University, Xiamen, Fujian 361102, China
}

Received 2 June 2020; Accepted (in revised version) 6 November 2020

\begin{abstract}
For flow simulations with complex geometries and structured grids, it is preferred for high-order difference schemes to achieve high accuracy. In order to achieve this goal, the satisfaction of free-stream preservation is necessary to reduce the numerical error in the numerical evaluation of grid metrics. For the linear upwind schemes with flux splitting the free-stream preserving property has been achieved in the early study [Q. Li et al., Commun. Comput. Phys., 22 (2017), pp. 64-94]. In the current paper, new series of nonlinear upwind-biased schemes through WENO interpolation will be proposed. In the new nonlinear schemes, the shock-capturing capability on distorted grids is achieved, which is unavailable for the aforementioned linear upwind schemes. By the inclusion of fluxes on the midpoints, the nonlinearity in the scheme is obtained through WENO interpolations, and the upwind-biased construction is acquired by choosing relevant grid stencils. New third- and fifth-order nonlinear schemes are developed and tested. Discussions are made among proposed schemes, alternative formulations of WENO and hybrid WCNS, in which a general formulation of center scheme with midpoint and nodes employed is obtained as a byproduct. Through the numerical tests, the proposed schemes can achieve the designed orders of accuracy and free-stream preservation property. In 1-D Sod and ShuOsher problems, the results are consistent with the theoretical predictions. In 2-D cases, the vortex preservation, supersonic inviscid flow around cylinder at $M_{\infty}=4$, Riemann problem, and shock-vortex interaction problems have been tested. More specifically, two types of grids are employed, i.e., the uniform/smooth grids and the randomized/locally-randomized grids. All schemes can get satisfactory results in uniform/smooth grids. On the randomized grids, most schemes have accomplished computations with reasonable accuracy, except the failure of one third-order scheme in Riemann problem and shock-vortex interaction. Overall, the proposed nonlinear schemes have the capability to solve flow problems on badly deformed grids, and the schemes can be used in the engineering applications.
\end{abstract}

*Corresponding author.

Email: qin-li@vip.tom.com (Q. Li) 
AMS subject classifications: 65M06

Key words: High-order difference scheme, upwind-biased scheme, WENO, interpolation, freestream preservation, hybrid WCNS.

\section{Introduction}

On developing high-order difference schemes, large advances have been achieved since the presence of WENO scheme series [1-13]. Besides WENO schemes, high-order compact schemes [14] were proposed as well with well-designed dispersion relations. Despite of abundant researches and outcomes, it is interesting to observe that high-order difference schemes are seldom applied for engineering complexities such as airplanes. In such situations, multi-blocked grids are inevitably used, the variation of grid topology usually happens, and meshes with locally bad quality might be encountered. For example, distorted grids would exist either inside blocks or on boundaries between them. Regarding the former one, Visbal and Gaitonde [15] showed that large errors may arise in computations, and similar conclusions were made in the subsequent studies [16,24]. Regarding the latter one, it was reported [18] that spurious oscillations would occur around the grid conjunctions. It is known that one of the causes would be the mismatches between evaluating grid metrics and solving conservative governing equations in curvilinear coordinate system.

In deriving conservative governing equations, theoretically zero-valued identities, namely metric identities, are introduced in partial differential operations [19]. However, the identities might not be null in practical computations [20]. In order to minimize such metric-caused errors, the following understandings are reached: (1) The grid metrics are suggested to adopt the conservative form rather than the expanded one [21,22]; (2) The consistent use of difference scheme in each coordinate direction should be applied to discretize the spatial derivatives in grid metrics and governing equations [15,23]. The key issue involved is the commutativity of difference operators [23], which was proved in [23] first and [24] later. With the help of commutativity, for an initially uniform flow, the flow will remain constant all the time, i.e., the satisfaction of the so-called free-stream preservation (FSP) property. Although FSP may be achieved by either linear central or upwind schemes, nonlinear mechanisms should become necessary to ensure numerical stability for the flow with possible discontinuity. In this regard, two methodologies have been employed for linear central schemes, i.e., filtering [15] and WENO interpolations [17,18]. For linear upwind schemes, however, the consistent use of certain scheme might invoke numerical instability because flow characteristics may contain both upwind and downwind components. Moreover, the flux is usually split into two parts with upwind discretization, the conventional implementation may make the schemes not to meet the requirement of the FSP property. 
In [25], Nonomura et al. developed the fifth- and sixth-order WENO schemes and re-arranged the original $\hat{f}_{j+1 / 2}$ in $\frac{\partial \hat{e}}{\partial \hat{\xi}}=\frac{\hat{f}_{j+1 / 2}-\hat{f}_{j-1 / 2}}{h_{s}}$ into a central consistent part plus two nonlinear dissipations in the splitting fluxes. In dissipative parts, a flux splitting-like operation was proposed to replace the standard splitting with a frozen treatment of grid metric. Combining the conservative metric derivation by Thomas et al. [21,22], the modified WENO schemes were shown to achieve FSP [25]. In [26], a detailed investigation was carried out in order to alleviate errors in metric evaluation for arbitrary linear upwind schemes with flux splitting. In the reference, the central scheme decomposition (CSD) was presented for metric evaluations, where the requirement for flux splitting to cancel out metric-evoked errors was derived. At the same time, a requirement for directionally consistent interpolation was proposed once the grid metrics on cell midpoints were involved in the schemes. Although the interpretation of an upwind scheme as a central scheme with additional dissipation part was used in above studies, the technique was used before. For example, the approach had been referred in TVD schemes [27] and high-order schemes [28-31]. In [26], some upwind linear schemes were proposed in the computations. However, they can only be applied in cases without shock waves. It is natural to further develop nonlinear upwind schemes with FSP in a general situation.

Based on the analyses in [26] and the introduction of nonlinearity through WENO interpolation [32] in [17, 18], new series of third-, fifth- and seventh-order WENO interpolation-based and upwind-biased schemes are proposed in this study, which are abbreviated as WENOIU for convenience. The schemes will be shown to achieve both shock capturing and FSP. The term "upwind-biased" means that with respect to the symmetric point (e.g., $x_{j}$ for schemes of $\left(\frac{\partial}{\partial x}\right)_{j}$ or $x_{j+1 / 2}$ for aforementioned $\hat{f}_{j+1 / 2}$ ), the number of upwind nodes and midpoints is one more than the number of the downwind ones. In [33], such form of scheme is shown to introduce less dissipation. The arrangement of the paper is as follows. Relevant studies are first reviewed in Section 2, then the construction of the third-, fifth-order WENOIU schemes are described there as well; in Section 3, the characteristics of the schemes are analyzed; in Section 4, the convergence property and FSP are discussed; in Section 5, validating examples are tested; at last, conclusions and discussions are given in Section 6.

\section{Nonlinear upwind-biased free-stream preserving scheme based on WENO interpolation}

\subsection{Governing equations and coordinate transformation}

3-D Euler equations are chosen for illustration and also for later validating computations. The equation in Cartesian coordinate system is

$$
\frac{\partial Q}{\partial t}+\frac{\partial E}{\partial x}+\frac{\partial F}{\partial y}+\frac{\partial G}{\partial z}=0
$$


where $Q=(\rho, \rho u, \rho v, \rho w, e)^{\mathrm{T}}, e=p /(\gamma-1)+1 / 2 \times \rho\left(u^{2}+v^{2}+w^{2}\right)$, and $\{E, F, G\}$ are inviscid flux vectors. In practical computations, Eq. (2.1) is usually transformed and solved in curvilinear coordinate system $(\xi, \eta, \zeta)$. The conversion from $(x, y, z)$ to $(\xi, \eta, \gamma)$ is derived through the chain rule such as: $\partial / \partial x=\xi_{x}(\partial / \partial \xi)+\eta_{x}(\partial / \partial \eta)+\zeta_{x}(\partial / \partial \zeta)$, and the transformed equation is

$$
\frac{\partial \hat{Q}}{\partial t}+\frac{\partial \hat{E}}{\partial \xi}+\frac{\partial \hat{F}}{\partial \eta}+\frac{\partial \hat{G}}{\partial \zeta}=0
$$

where

$$
\begin{array}{ll}
\hat{Q}=J^{-1} Q & \text { with } J^{-1}=\left|\frac{\partial(x, y, z)}{\partial(\xi, \eta, \zeta)}\right| \\
\hat{E}=\left(\hat{\xi}_{x} E+\hat{\zeta}_{y} F+\hat{\zeta}_{z} G\right) & \text { with } \quad \hat{\xi}_{x}=J^{-1} \xi_{x}
\end{array}
$$

and etc. In the derivation, Leibniz formula is freely used and the theoretically zero-valued term $\left(\hat{E} \cdot I_{x}+\hat{F} \cdot I_{y}+\hat{G} \cdot I_{z}\right)$ has been dropped. In the dropped term, the so-called metric identity $I_{x_{i}}$ is defined as $I_{x_{i}}=\left(\hat{\zeta}_{x_{i}}\right)_{\xi}+\left(\hat{\eta}_{x_{i}}\right)_{\eta}+\left(\hat{\zeta}_{x_{i}}\right)_{\zeta^{\prime}}$, where $x_{i}$ denotes $x, y$ or $z$.

\subsection{Reviews on difference schemes to achieve free-stream preservation}

(1) Causes of violation of free-stream preservation.

From Eq. (2.2), it is commonplace that when the uniform flow is imposed as the initial condition in a field without bodies (abbreviated as free-stream condition below), $\hat{Q}$ would keep constant theoretically. However, $\hat{Q}$ might change in practical computations, and the main causes lie in the use of inappropriate formula of grid metric [21,22] and inconsistent implementation of partial differential operators in Eq. (2.2) [15,23]. Regarding the latter, although derivatives such as $\frac{\partial}{\partial \xi}$ in $\frac{\partial \hat{E}}{\partial \tilde{\xi}}$ and $\hat{\eta}_{x_{i}}$ of $\frac{\partial \hat{F}}{\partial \eta}$ are mathematically indistinguishable, their concrete implementations might be numerically different. Supposing the following conservative forms of grid metric are employed and free-stream condition are further casted, the spatial derivatives in Eq. (2.2) will be simplified and turned into mixed partial derivatives such as $\frac{\partial^{2} x_{i}}{\partial \xi \partial \eta}$ and $\frac{\partial^{2} x_{i}}{\partial \eta \partial \xi}$. As just mentioned, the derivatives might be numerically different, or the so-called commutativity property would be lost. The loss of commutativity will yield non-zero values of $I_{x_{i}}$, which results in the violation of FSP at last. It is proven in $[23,24]$ that when subsequent "conservative" formulae of grid metrics would be chosen and spatial derivatives to discretize metrics and fluxes in Eq. (2.2) would keep consistent in each coordinate direction, FSP could be achieved when solving Eq. (2.2). Concrete implementations will be reviewed next.

(2) Conservative formulae of grid metrics.

References $[23,24]$ have shown that under free-stream condition, the following two forms of grid metric would make spatial derivatives in Eq. (2.2) degenerate into terms 
like $\left(\frac{\partial^{2} x_{i}}{\partial \tilde{y}^{2} \eta}-\frac{\partial^{2} x_{i}}{\partial \eta \partial \xi}\right)$, which would be cancelled out if the commutativity holds. From [21], the conservative forms of grid metrics are:

$$
\begin{array}{lll}
\hat{\xi}_{x}=\left(y_{\eta} \cdot z\right)_{\zeta}-\left(y_{\zeta} \cdot z\right)_{\eta^{\prime}} & \hat{\xi}_{y}=\left(z_{\eta} \cdot x\right)_{\zeta}-\left(z_{\zeta} \cdot x\right)_{\eta^{\prime}} & \hat{\xi}_{z}=\left(x_{\eta} \cdot y\right)_{\zeta}-\left(x_{\zeta} \cdot y\right)_{\eta^{\prime}} \\
\hat{\eta}_{x}=\left(y_{\zeta} \cdot z\right)_{\xi}-\left(y_{\xi} \cdot z\right)_{\zeta^{\prime}}, & \hat{\eta}_{y}=\left(z_{\zeta} \cdot x\right)_{\xi}-\left(z_{\xi} \cdot x\right)_{\zeta^{\prime}} & \hat{\eta}_{z}=\left(x_{\zeta} \cdot y\right)_{\xi}-\left(x_{\xi} \cdot y\right)_{\zeta^{\prime}} \\
\hat{\zeta}_{x}=\left(y_{\xi} \cdot z\right)_{\eta}-\left(y_{\eta} \cdot z\right)_{\xi^{\prime}} & \hat{\zeta}_{y}=\left(z_{\xi} \cdot x\right)_{\eta}-\left(z_{\eta} \cdot x\right)_{\xi^{\prime}} & \hat{\zeta}_{z}=\left(x_{\xi} \cdot y\right)_{\eta}-\left(x_{\eta} \cdot y\right)_{\xi} .
\end{array}
$$

Furthermore, the symmetric conservative forms [22] are

$$
\begin{array}{ll}
\hat{\xi}_{x}=\frac{1}{2}\left[\begin{array}{c}
\left(y_{\eta} \cdot z\right)_{\zeta}-\left(y_{\zeta} \cdot z\right)_{\eta} \\
+\left(z_{\zeta} \cdot y\right)_{\eta}-\left(z_{\eta} \cdot y\right)_{\zeta}
\end{array}\right], & \hat{\xi}_{y}=\frac{1}{2}\left[\begin{array}{c}
\left(z_{\eta} \cdot x\right)_{\zeta}-\left(z_{\zeta} \cdot x\right)_{\eta} \\
+\left(x_{\zeta} \cdot z\right)_{\eta}-\left(x_{\eta} \cdot z\right)_{\zeta}
\end{array}\right], \\
\hat{\xi}_{z}=\frac{1}{2}\left[\begin{array}{c}
\left(x_{\eta} \cdot y\right)_{\zeta}-\left(x_{\zeta} \cdot y\right)_{\eta} \\
+\left(y_{\zeta} \cdot x\right)_{\eta}-\left(y_{\eta} \cdot x\right)_{\zeta}
\end{array}\right], & \hat{\eta}_{x}=\frac{1}{2}\left[\begin{array}{c}
\left(y_{\zeta} \cdot z\right)_{\xi}-\left(y_{\xi} \cdot z\right)_{\zeta} \\
+\left(z_{\xi} \cdot y\right)_{\zeta}-\left(z_{\zeta} \cdot y\right)_{\xi}
\end{array}\right], \\
\hat{\eta}_{y}=\frac{1}{2}\left[\begin{array}{c}
\left(z_{\zeta} \cdot x\right)_{\xi}-\left(z_{\xi} \cdot x\right)_{\zeta} \\
+\left(x_{\xi} \cdot z\right)_{\zeta}-\left(x_{\zeta} \cdot z\right)_{\xi}
\end{array}\right], & \hat{\eta}_{z}=\frac{1}{2}\left[\begin{array}{c}
\left(x_{\zeta} \cdot y\right)_{\xi}-\left(x_{\xi} \cdot y\right)_{\zeta} \\
+\left(y_{\xi} \cdot x\right)_{\zeta}-\left(y_{\zeta} \cdot x\right)_{\xi}
\end{array}\right], \\
\hat{\zeta}_{x}=\frac{1}{2}\left[\begin{array}{c}
\left(y_{\xi} \cdot z\right)_{\eta}-\left(y_{\eta} \cdot z\right)_{\xi} \\
+\left(z_{\eta} \cdot y\right)_{\xi}-\left(z_{\xi} \cdot y\right)_{\eta}
\end{array}\right], & \hat{\zeta}_{y}=\frac{1}{2}\left[\begin{array}{c}
\left(z_{\xi} \cdot x\right)_{\eta}-\left(z_{\eta} \cdot x\right)_{\xi} \\
+\left(x_{\eta} \cdot z\right)_{\xi}-\left(x_{\xi} \cdot z\right)_{\eta}
\end{array}\right], \\
\hat{\zeta}_{z}=\frac{1}{2}\left[\begin{array}{c}
\left(x_{\xi} \cdot y\right)_{\eta}-\left(x_{\eta} \cdot y\right)_{\xi} \\
+\left(y_{\eta} \cdot x\right)_{\xi}-\left(y_{\xi} \cdot x\right)_{\eta}
\end{array}\right] . &
\end{array}
$$

Although not theoretically being related to metric-caused errors, the evaluations of Jacobian are suggested as [35]

$$
J^{-1}=\frac{1}{3}\left[\begin{array}{c}
\hat{\xi}_{x} \cdot x_{\xi}+\hat{\zeta}_{y} \cdot y_{\xi}+\hat{\zeta}_{z} \cdot z_{\xi} \\
+\hat{\eta}_{x} \cdot x_{h}+\hat{\eta}_{y} \cdot y_{\eta}+\hat{\eta}_{z} \cdot z_{\eta} \\
+\hat{\zeta}_{x} \cdot x_{\zeta}+\hat{\zeta}_{y} \cdot y_{\zeta}+\hat{\zeta}_{z} \cdot z_{\zeta}
\end{array}\right]
$$

or

$$
J^{-1}=\frac{1}{3}\left[\begin{array}{c}
\left(\hat{\xi}_{x} \cdot x\right)_{\xi}+\left(\hat{\xi}_{y} \cdot y\right)_{\xi}+\left(\hat{\xi}_{z} \cdot z\right)_{\xi} \\
+\left(\hat{\eta}_{x} \cdot x\right)_{\eta}+\left(\hat{\eta}_{y} \cdot y\right)_{\eta}+\left(\hat{\eta}_{z} \cdot z\right)_{\eta} \\
+\left(\hat{\zeta}_{x} \cdot x\right)_{\zeta}+\left(\hat{\zeta}_{y} \cdot y\right)_{\zeta}+\left(\hat{\zeta}_{z} \cdot z\right)_{\zeta}
\end{array}\right] .
$$

Besides, the derivations of grid metrics in $J^{-1}$ are suggested to use Eq. (2.3) or (2.4).

(3) Achieving free-stream preservation by linear schemes with flux splitting.

As mentioned above, the cancellation such as $\frac{\partial^{2} x_{i}}{\partial \xi^{\xi} \partial \eta}-\frac{\partial^{2} x_{i}}{\partial \eta \partial \xi}$ requires the commutativity of operators to be established, which entails the consistent implementation of $\frac{\partial}{\partial \xi}$ and $\frac{\partial}{\partial \eta}[15,23]$. In Eq. (2.2), the appearance of operator like $\frac{\partial}{\partial \xi}$ has two origins: the one 
comes from the flux derivative (e.g., $\frac{\partial \hat{E}}{\partial \xi}$ ) and the other comes from the metric evaluation. Regarding the former, there are two basic types of discretization when flux splitting is considered, i.e., the central and upwind scheme. For central schemes, the presence of flux splitting will not bring about difficulty to the consistent implementation because the scheme can keep constant during discretization. Moreover, when midpoints are included in schemes, WENO interpolation can be applied to derive function values on them. By this means, the flux splitting can be introduced naturally and necessary dissipations will be invoked when shock waves are encountered $[17,18]$. However, the concrete form of upwind scheme would vary depending on the windward characteristics of the splitting flux, and therefore the consistent implementation could not be easily realized.

In [25], a method was proposed by re-arranging the canonical fifth-order WENO schemes (WENO5) into one consistent central part plus two nonlinear dissipation parts. In the latter metric linearization is used for flux splitting; besides, the central part is used for metric evaluation. In [26], investigations are carried out independently as well. It reads that [26], if a $r$-th-order linear upwind scheme $\delta^{+}$for $\partial f$ at $x_{j}$ is considered where $\partial$ denotes the differential operator for simplicity and $\delta$ denotes its difference approximation, its downwind counterpart $\delta^{-}$will be defined symmetrically. Hence a central difference scheme $\delta^{c,(1)}$ can be derived as

$$
\left(\delta^{c,(1)} f\right)=\left[\left(\delta^{+} f_{j}\right)+\left(\delta^{-} f_{j}\right)\right] / 2
$$

From the equation, another central discretization of $\partial^{(2\lfloor r / 2\rfloor+2)} f$ naturally comes out through

$$
\delta^{c,(2\lfloor r / 2\rfloor+2)}=\left(\delta^{c,(1)} f\right)_{j}-\left(\delta^{+} f\right)_{j^{\prime}}
$$

where the number in superscript bracket such as $2\lfloor r / 2\rfloor+2$ denotes the derivative order. By way of the above procedure, the following decompositions are straightforward

$$
\left\{\begin{array}{l}
\left(\delta^{+} f_{j}\right)=\left(\delta^{c,(1)} f\right)-\left(\delta^{c,(2\lfloor r / 2\rfloor+2)} f\right), \\
\left(\delta^{-} f_{j}\right)=\left(\delta^{c,(1)} f\right)+\left(\delta^{c,(2\lfloor r / 2\rfloor+2)} f\right),
\end{array}\right.
$$

which is referred as central scheme decomposition in [26]. Similar practice has been observed in previous studies [27-31], and the decomposition of linear counterpart of WENO5 as two central schemes was also available there.

In [26], the derived $\delta^{c,(1)}$ is used to discretize derivatives in grid metrics, while the original $\delta^{+}$and $\delta^{-}$will be normally used in discretizing flux derivatives as usual. Besides the above manipulations regarding spatial discretization, another requirement regarding the flux splitting is proposed in [26], which is necessary for the achievement of FSP. Considering that the flux splitting with the form as

$$
\hat{E}^{ \pm}=\frac{1}{2}(\hat{E} \pm \hat{A} \cdot Q) \quad \text { or } \quad \hat{E}^{ \pm}=\frac{1}{2}\left(\hat{E} \pm \hat{E}_{r e f}\right),
$$


one sufficient condition is: when the uniform flow condition is imposed, $\hat{A}$ and $\hat{E}_{\text {ref }}$ should be locally constant at least over the dependent grid stencil of $\delta^{c,(2\lfloor r / 2\rfloor+2)}$. Details of the analysis can be found in [26], and the form of $\hat{A}$ used in this paper is:

$$
\hat{A}=\alpha \cdot \operatorname{MAX}(\hat{U}+c|\hat{k}|),
$$

where $\alpha=1.1 \sim 1.2, \hat{U}=\hat{k}_{x} u+\hat{k}_{y} v+\hat{k}_{z} w$ and $\hat{k}=\left(\hat{k}_{x}^{2}+\hat{k}_{y}^{2}+\hat{k}_{z}^{2}\right)^{1 / 2}$ with $k$ denoting certain curvilinear coordinate, and where MAX runs over the whole range of the $k$ direction. Analyses were made and validations were given in [26] to show that above manipulations would make arbitrary linear scheme to achieve FSP with the presence of flux splitting.

(4) Requirements for interpolations when cell midpoints are included in difference schemes.

When cell midpoints are employed in difference schemes, two types of interpolations will be invoked in order to derive unknowns on them. The first type regards the derivation of function values on midpoints. In this aspect, WENO interpolations can be applied, e.g., WCNS series schemes [17] are established in this way. In the interpolations, the following types of variable are observed to use, i.e., the primitive, conservative, characteristic variables [36] and the flux [37]. For WCNS, the first three types have been tested to achieve FSP $[17,18]$ on distorted grids such as randomized ones. For the last variable type, successful computations have been made on typical 2-D problems on uniform Cartesian grids; however, FSP may not be guaranteed on distorted grids because grid metrics are included in flux during interpolation. Hence, in order to achieve FSP, the first three types of variables are suggested to use.

The second type of interpolation regards grid metrics at cell midpoints. Required by difference schemes $[17,18]$ including midpoints, grid metrics therein should be acquired by interpolation. The most convenient way for acquisition is that: evaluate the function values on nodes firstly by following the instructions in (2) and (3), then interpolate them to the midpoints. In [26], a sufficient requirement to achieve FSP has been proposed as: the consistent linear interpolation should be casted in each coordinate direction (so-called directionally consistent interpolation) in the evaluation of metrics and metrics in fluxes at midpoints; besides, the interpolations could be different in different directions. In [26], a proof of this requirement has been given and numerical validations have been provided. Specifically, a fourth-order interpolation is employed in this study as:

$$
f_{i+1 / 2} \approx \frac{1}{16}\left(-f_{i-1}+9 f_{i}+9 f_{i+1}-f_{i+2}\right) .
$$

(5) Short summary.

By means of the above methodologies, the linear upwind schemes with flux splitting can achieve FSP. However, such schemes can only be applicable for problems without shock waves because of the linearity. Although the method in [25] is nonlinear, it poses specific implementation of flux splitting, which is almost the same as Lax-Friedrichs 
scheme with the little difference on the use of linearized grid metrics. On the one hand Lax-Friedrichs scheme is found to be dissipative, on the other hand recent schemes such as AUSM series and etc. cannot be applied in the method. To overcome the above insufficiencies, new upwind-biased nonlinear schemes are proposed next with the achievement of shock-capturing together with FSP.

\subsection{WENO interpolation-based and upwind-biased scheme with free-stream preservation}

Based on the understandings in Section 2.2, new series of third-, fifth- and seventh-order upwind-biased conservative schemes (WENOIU) are proposed with the nonlinearity introduced by WENO interpolation. In the schemes, the number of grid points (nodes and midpoints) in upwind direction is one more than that in downwind direction with respect to $x_{j+1 / 2}$. With the help of nonlinearity, WENOIU schemes will not only achieve FSP, but also can solve problems with shock waves even on randomized grids. Such performance implies application capability for engineering.

(1) Construction of difference schemes.

Consider the following one-dimensional hyperbolic conservative law for illustration,

$$
u_{t}+f(u)_{x}=0
$$

Supposing the grid is discretized as $x_{i}=i \cdot \Delta x$, where $\Delta x$ is the uniform space interval, the semi-discretized conservative formula at $x_{j}$ holds

$$
\left(u_{t}\right)_{j}=-\left(h_{j+1 / 2}-h_{j-1 / 2}\right) / \Delta x,
$$

where $h(u)$ is implicitly defined by

$$
f(x)=\frac{1}{\Delta x} \int_{x-\Delta x / 2}^{x+\Delta x / 2} h\left(x^{\prime}\right) d x^{\prime} .
$$

The formula in [34] to derive $h(u)$ is chosen here to further develop WENOIU schemes as

$$
\begin{gathered}
h_{j+1 / 2}=f_{j+1 / 2}-\frac{1}{24} \Delta x^{2}\left(\frac{\partial^{2} f}{\partial x^{2}}\right)_{j+1 / 2}+\frac{7}{5760} \Delta x^{4}\left(\frac{\partial^{4} f}{\partial x^{4}}\right)_{j+1 / 2} \\
-\frac{31}{967680} \Delta x^{6}\left(\frac{\partial^{6} f}{\partial x^{6}}\right)_{j+1 / 2}+\mathcal{O}\left(\Delta x^{8}\right) .
\end{gathered}
$$

Considering similar difference schemes involving cell midpoints such as that in [14], high-order upwind schemes are available through discretizing $\left(\frac{\partial^{n} f}{\partial x^{n}}\right)_{j+1 / 2}$.

When derivatives such as $\left(\frac{\partial^{n} f}{\partial x^{n}}\right)_{j+1 / 2}$ in Eq. (2.12) are discretized linearly, combinations of $f$ on grid points would be used. Two types of grid points can be employed, i.e., 
nodes and cell midpoints. As previously mentioned, when $f$ on midpoints are involved, interpolations should be invoked. If WENO interpolation is further adopted, the nonlinearity will be introduced accordingly. By this way, on the one hand the linear form of an upwind difference scheme can keep unchanged seemingly, which is favored by the requirement of FSP [26], on the other hand the nonlinearity would therefore be implemented. By this means, the third-, fifth- and seventh-order schemes are constructed with various midpoints included.

Prior to further analysis, additional narration will be made regarding flux splitting. When flux splitting is considered, the flux at cell nodes and midpoints will be split as $f=f^{+}+f^{-}$, and so does $h_{j+1 / 2}$ (i.e., $h_{j+1 / 2}=h^{+}{ }_{j+1 / 2}+h^{-}{ }_{j+1 / 2}$ ). Accordingly, the upwind scheme will be related with corresponding splitting flux. In the following, the construction of upwind-biased schemes $\left(\hat{f}_{j+1 / 2}^{+}\right)$is illustrated by the one regarding $f^{+}$, and for simplicity the superscript + will be dropped also. The counterpart of the scheme regarding $f^{-}$, i.e., $\hat{f}_{j+1 / 2}^{-}$will have the symmetric form of $\hat{f}_{j+1 / 2}^{+}$with respect to $x_{j+1 / 2}$ as usual [2].

(2) Third-order WENOIU schemes (WENOIU3).

Considering Eq. (2.12), it is obvious that only $\left(\frac{\partial^{2} f}{\partial x^{2}}\right)_{j+1 / 2}$ needs to be discretized, and corresponding schemes are categorized according to the number of midpoints included.

(a) Scheme with only one midpoint $x_{j+1 / 2}$ included.

Using Taylor series expansion, the approximation of $h_{j+1 / 2}$ or $\hat{f}_{j+1 / 2}$ can be obtained as

$$
\hat{f}_{j+1 / 2}=f_{j+1 / 2}+\frac{1}{24}\left(-f_{j-1}+2 f_{j}-f_{j+1}\right) .
$$

Considering the upwind-biased nature, the number of nodes to approximate $h_{j+1 / 2}$ should be $2 m+1$ with $m \geq 1$, therefore the total number of points including that of $x_{j+1 / 2}$ will be $2 m+2$. Hence, the above scheme should have one free parameter. Based on the understanding, the more general third-order form can be derived as

$$
\hat{f}_{j+1 / 2}=\alpha f_{j+1 / 2}+\left(\frac{1}{8} \alpha-\frac{1}{6}\right) f_{j-1}+\left(-\frac{3}{4} \alpha+\frac{5}{6}\right) f_{j}+\left(-\frac{3}{8} \alpha+\frac{1}{3}\right) f_{j+1} .
$$

When $\alpha=8 / 9$ in the equation, the non-upwind-biased scheme with the minimum points can be obtained as

$$
\hat{f}_{j+1 / 2}=\frac{8}{9} f_{j+1 / 2}-\frac{1}{18} f_{j-1}+\frac{1}{6} f_{j} .
$$

The accuracy relation of Eq. (2.14) is:

$$
\begin{aligned}
f_{j+1 / 2}- & \frac{1}{24} \Delta x^{2}\left(\frac{\partial^{2} f}{\partial x^{2}}\right)_{j+1 / 2}+\left(\frac{1}{16} \alpha-\frac{1}{12}\right) \Delta x^{3}\left(\frac{\partial^{3} f}{\partial x^{3}}\right)_{j+1 / 2} \\
+ & \left(\frac{3}{128} \alpha-\frac{37}{1152}\right) \Delta x^{4}\left(\frac{\partial^{4} f}{\partial x^{4}}\right)_{j+1 / 2}+\mathcal{O}\left(\Delta x^{5}\right) .
\end{aligned}
$$


Taking the order and number of midpoints as indicators, the scheme by Eq. (2.14) is referred as WENOIU3-1MP. The general terminology is like "WENOIUn-mMP", where $n$ denotes the scheme order and $m$ denotes the midpoint number. In this study, only Eq. (2.13) is tested. The use of Eq. (2.14) will be further discussed in other investigations.

According to Eq. (2.7), $\delta^{c,(1)}$ of Eq. (2.13), which is derived to compute grid metrics, is

$$
\delta^{c,(1)} \mathbf{x}=\frac{1}{\Delta x}\left[\left(\mathbf{x}_{j+1 / 2}-\mathbf{x}_{j-1 / 2}\right)+\frac{1}{48}\left(-\mathbf{x}_{j+2}+2 \mathbf{x}_{j+1}-2 \mathbf{x}_{j-1}+\mathbf{x}_{j-2}\right)\right],
$$

which is a fourth-order central difference scheme and where $\mathbf{x}$ denotes coordinate $x, y$ or $z$.

(b) Scheme with two midpoints included (WENOIU3-2MP).

Similarly, corresponding scheme can be derived as

$$
\hat{f}_{j+1 / 2}=f_{j+1 / 2}+\frac{1}{6}\left(-f_{j-1 / 2}+2 f_{j}-f_{j+1 / 2}\right) .
$$

Its accuracy relation is

$$
f_{j+1 / 2}-\frac{1}{24} \Delta x^{2}\left(\frac{\partial^{2} f}{\partial x^{2}}\right)_{j+1 / 2}+\frac{1}{48} \Delta x^{3}\left(\frac{\partial^{3} f}{\partial x^{3}}\right)_{j+1 / 2}-\frac{7}{1152} \Delta x^{4}\left(\frac{\partial^{4} f}{\partial x^{4}}\right)_{j+1 / 2}+\mathcal{O}\left(\Delta x^{5}\right) .
$$

Similarly, the scheme by Eq. (2.17) is referred as WENOIU3-2MP. It is worthwhile to mention that the overall scheme for $(\partial f / \partial x)_{j}$ from Eq. (2.17) has been referred in [26].

According to Eq. (2.7), the fourth-order $\delta^{c,(1)}$ of Eq. (2.17) to compute grid metrics is

$$
\delta^{c,(1)} \mathbf{x}=\frac{1}{12 \Delta x}\left[2\left(\mathbf{x}_{j+1}-\mathbf{x}_{j-1}\right)+\left(-\mathbf{x}_{j+3 / 2}+11 \mathbf{x}_{j+1 / 2}-11 \mathbf{x}_{j-1 / 2}+\mathbf{x}_{j-3 / 2}\right)\right],
$$

where $\mathbf{x}$ denotes coordinates as before.

(c) WENO interpolation [32].

In Eqs. (2.13)-(2.15) and (2.17), unknown fluxes on midpoints should be acquired by interpolation of $u$, and through which the implementation of scheme could be accomplished. From [32], the third-order WENO interpolation is

$$
u_{j+1 / 2}=\sum_{k=0}^{r-1} \omega_{k} p_{k}^{r}
$$


Table 1: Coefficients $b_{k, l}^{r}$ and $C_{k}^{r}$ in interpolation schemes.

\begin{tabular}{||c|c|c|ccc||}
\hline \multirow{2}{*}{$r$} & \multirow{2}{*}{$k$} & \multirow{2}{*}{$C_{k}^{r}$} & \multicolumn{3}{|c||}{$b_{k, l}^{r}$} \\
\cline { 4 - 6 } & & & $l=0$ & $l=1$ & $l=2$ \\
\hline \multirow{2}{*}{2} & 0 & $1 / 4$ & $-1 / 2$ & $3 / 2$ & - \\
\cline { 2 - 6 } & 1 & $3 / 4$ & $1 / 2$ & $1 / 2$ & - \\
\hline \multirow{3}{*}{3} & 0 & $1 / 16$ & $3 / 8$ & $-10 / 8$ & $15 / 8$ \\
\cline { 2 - 6 } & 1 & $10 / 16$ & $-1 / 8$ & $6 / 8$ & $3 / 8$ \\
\cline { 2 - 6 } & 2 & $5 / 16$ & $3 / 8$ & $6 / 8$ & $-1 / 8$ \\
\hline
\end{tabular}

where $r$ denotes the grid number of stencil of candidate scheme $p_{k}^{r}$ or $r=2$ here. The form of $p_{k}^{r}$ is

$$
p_{k}^{r}=\sum_{l=0}^{r-1} b_{k, l}^{r} u_{j-r+k+l+1}
$$

The coefficients $b_{k, l}^{r}$ in Eq. (2.20) are given in Table 1 [32]. $\omega_{k}$ is the nonlinear weight derived from its linear counterpart $C_{k}^{r}$ which is shown in Table 1 as well. The canonical weighting procedure is

$$
\omega_{k}=\alpha_{k} / \sum_{l=0}^{r-1} \alpha_{l}
$$

where

$$
\alpha_{k}=C_{k}^{r} /\left(\varepsilon+I S_{k}\right)^{2}
$$

and from [2], $I S_{k}$ is suggested as

$$
I S_{k}=\left(u_{j+k}-u_{j+k-1}\right)^{2} .
$$

It is known that at critical points, order degradation of standard WENO interpolation will occur. If such occurrence is quite concerned, methods such as the mapping function [5] can be applied, and a specific one named as the piecewise polynomial mapping function (abbreviated as PPM) [7] is chosen in this study. In case of thirdorder schemes, the second-order PPM can be used with the form as

$$
g(\omega)= \begin{cases}C_{k}\left[1-\left(\frac{\omega}{C_{k}}-1\right)^{2}\right], & \omega \leq C_{k}, \\ C_{k}-\frac{1}{C_{k}-1}\left(\omega-C_{k}\right)^{2}, & \omega>C_{k} .\end{cases}
$$

Besides, some special treatment should be imposed regarding $I S_{k}$, which will be explained later in Section 4.1. For clarity, the remedy is summarized as: after initial evaluation of $\omega$ by Eqs. (2.21)-(2.22), Eq. (2.24) is invoked for $\omega$; afterwards the 
normalization is carried out on mapped values to yield the final $\omega$. From [5,7], $\varepsilon$ can have a very small quantity such as $10^{-40}$ when Eq. (2.24) is used, otherwise it takes the value $10^{-6}$ in the computation.

At last, it can be testified that Eq. (2.14) and Eq. (2.17) are all the possible upwindbiased schemes which contain midpoints.

(d) Flux splitting.

In (3) in Section 2.2, the flux splitting method is described regarding upwind schemes, which will be applied to WENOIU3 and WENOIU5 next accordingly. One particularity exists regarding WENOIU3-1MP, i.e., $f_{j+1 / 2}$ takes a special role analogous to a central scheme locally. Considering discussions in (3) in Section 2.2 and conclusions in $[24,26]$, the flux splitting of $f_{j+1 / 2}$ can employ either aforementioned method or other schemes such as Steger-Warming scheme, AUSM series and etc. The split scheme of the rest fluxes in WENOIU3-1MP should still take that in (3) in Section 2.2.

(3) Fifth-order WENOIU schemes (WENOIU5).

From Eq. (2.12), both $\left(\frac{\partial^{2} f}{\partial x^{2}}\right)_{j+1 / 2}$ and $\left(\frac{\partial^{4} f}{\partial x^{4}}\right)_{j+1 / 2}$ need to be discretized, and corresponding schemes are categorized according to the number of midpoints included as well.

(a) Scheme with only one midpoint $x_{j+1 / 2}$ included (WENOIU5-1MP).

By discretizing the second- and fourth-order derivatives, the following fifth-order scheme can be obtained as

$$
\hat{f}_{j+1 / 2}=f_{j+1 / 2}+\frac{19}{1920} f_{j-2}-\frac{29}{480} f_{j-1}+\frac{77}{960} f_{j}-\frac{3}{160} f_{j+1}-\frac{7}{640} f_{j+2} .
$$

As mentioned before, the more general form which has one free parameter is

$$
\begin{gathered}
\hat{f}_{j+1 / 2}=\alpha f_{j+1 / 2}+\left(-\frac{3}{128} \alpha+\frac{1}{30}\right) f_{j-2}+\left(\frac{5}{32} \alpha-\frac{13}{60}\right) f_{j-1}+\left(-\frac{45}{64} \alpha+\frac{47}{60}\right) f_{j} \\
+\left(-\frac{15}{32} \alpha+\frac{9}{20}\right) f_{j+1}+\left(\frac{5}{128} \alpha-\frac{1}{20}\right) f_{j+2}
\end{gathered}
$$

When $\alpha=32 / 25$, the non-upwind-biased scheme with one point less is obtained as:

$$
\hat{f}_{j+1 / 2}=\frac{32}{25} f_{j+1 / 2}+\frac{1}{300} f_{j-2}-\frac{1}{60} f_{j-1}+\frac{7189}{24000} f_{j}-\frac{3}{20} f_{j+1} .
$$

The accuracy relation of Eq. (2.26) can be derived as

$$
\begin{aligned}
f_{j+1 / 2} & -\frac{1}{24} \Delta x^{2}\left(\frac{\partial^{2} f}{\partial x^{2}}\right)_{j+1 / 2}+\frac{7}{5760} \Delta x^{4}\left(\frac{\partial^{4} f}{\partial x^{4}}\right)_{j+1 / 2}+\frac{1}{3840}(45 \alpha-64) \Delta x^{5}\left(\frac{\partial^{5} f}{\partial x^{5}}\right)_{j+1 / 2} \\
& -\frac{1}{138240}(675 \alpha-983) \Delta x^{6}\left(\frac{\partial^{6} f}{\partial x^{6}}\right)_{j+1 / 2}+\mathcal{O}\left(\Delta x^{7}\right) .
\end{aligned}
$$


The scheme by Eq. (2.26) is referred as WENOIU5-1MP. Similarly, only Eq. (2.25) is tested in current study.

According to Eq. (2.7), the sixth-order $\delta^{c,(1)}$ of Eq. (2.25) to compute grid metrics is

$$
\begin{array}{r}
\delta^{c,(1)} \mathbf{x}=\frac{1}{\Delta x}\left(\mathbf{x}_{j+1 / 2}-\mathbf{x}_{j-1 / 2}\right)+\frac{1}{\Delta x}\left[\frac{19}{3840}\left(\mathbf{x}_{j+3}-\mathbf{x}_{j-3}\right)\right. \\
\left.-\frac{13}{320}\left(\mathbf{x}_{j+2}-\mathbf{x}_{j-2}\right)+\frac{17}{256}\left(\mathbf{x}_{j+1}-\mathbf{x}_{j-1}\right)\right],
\end{array}
$$

where $\mathbf{x}$ denotes coordinates as before.

(b) Scheme with two midpoints included.

The scheme can be derived as

$$
\hat{f}_{j+1 / 2}=f_{j+1 / 2}+\frac{1}{30}\left[\left(-4 f_{j+1}+f_{j}+f_{j-1}\right)+\left(6 f_{j+1 / 2}-f_{j-1 / 2}\right)\right]
$$

with the accuracy relation as

$$
\begin{aligned}
& f_{j+1 / 2}-\frac{1}{24} \Delta x^{2}\left(\frac{\partial^{2} f}{\partial x^{2}}\right)_{j+1 / 2}+\frac{7}{5760} \Delta x^{4}\left(\frac{\partial^{4} f}{\partial x^{4}}\right)_{j+1 / 2} \\
& -\frac{1}{960} \Delta x^{5}\left(\frac{\partial^{5} f}{\partial x^{5}}\right)_{j+1 / 2}+\frac{47}{138240} \Delta x^{6}\left(\frac{\partial^{6} f}{\partial x^{6}}\right)_{j+1 / 2}+\mathcal{O}\left(\Delta x^{7}\right) .
\end{aligned}
$$

Similarly, the scheme by Eq. (2.29) is denoted as WENOIU5-2MP. Corresponding sixth-order $\delta^{c,(1)}$ by Eq. (2.7) to compute grid metrics is

$$
\begin{aligned}
\delta^{c,(1)} \mathbf{x}= & \frac{1}{60 \Delta x}\left(\mathbf{x}_{i+2}-4 \mathbf{x}_{j+1}+4 \mathbf{x}_{j-1}-\mathbf{x}_{j-2}\right) \\
& +\frac{1}{15 \Delta x}\left(-\mathbf{x}_{j+3 / 2}+19 \mathbf{x}_{j+1 / 2}-19 \mathbf{x}_{j-1 / 2}+\mathbf{x}_{j-3 / 2}\right)
\end{aligned}
$$

(c) WENO interpolation [32].

The function values at cell midpoints are derived through WENO interpolation as that in "(2)" of this section but with $r=3$. The coefficients in Eq. (2.20) are shown in Table 1 as well. The smoothness indicators in Eq. (2.22) are [32]

$$
\left\{\begin{array}{l}
I S_{0}=\frac{1}{3}\left(4 u_{j-2}^{2}-19 u_{j-2} u_{j-1}+25 u_{j-1}^{2}+11 u_{j-2} u_{j}-31 u_{j-1} u_{j}+10 u_{j}^{2}\right) \\
I S_{1}=\frac{1}{3}\left(4 u_{j-1}^{2}-13 u_{j-1} u_{j}+13 u_{j}^{2}+5 u_{j-1} u_{j+1}-13 u_{j} u_{j+1}+4 u_{j+1}^{2}\right) \\
I S_{2}=\frac{1}{3}\left(10 u_{j}^{2}-31 u_{j} u_{j+1}+25 u_{j+1}^{2}+11 u_{j} u_{j+2}-19 u_{j+1} u_{j+2}+4 u_{j+2}^{2}\right)
\end{array} .\right.
$$


Taylor series expansion of Eq. (2.31) is:

$$
\left(\frac{\partial u}{\partial x}\right)_{j}^{2} \Delta x^{2}+\left(a \frac{\partial u}{\partial x} \frac{\partial^{3} u}{\partial x^{3}}+\frac{13}{12}\left(\frac{\partial^{2} u}{\partial x^{2}}\right)^{2}\right)_{j} \Delta x^{4}+\mathcal{O}\left(\Delta x^{5}\right),
$$

where $\alpha=-2 / 3,1 / 3$ and $-2 / 3$, respectively. When $(\partial u / \partial x)_{j}=0$, the residual $\mathcal{O}\left(\Delta x^{5}\right)$ is not zero usually, therefore Eq. (2.31) still suffers from order degradation at critical points. If such situation is quite concerned, the mapping function of [5] or the following third-order PPM can be used [7]

$$
g(\omega)= \begin{cases}C_{k}\left[1+\left(\frac{\omega}{C_{k}}-1\right)^{3}\right], & \omega \leq C_{k} \\ C_{k}+\left(\frac{1}{C_{k}-1}\right)^{2}\left(\omega-C_{k}\right)^{3}, & \omega>C_{k} .\end{cases}
$$

At last, it can be testified as well that Eq. (2.26) and Eq. (2.29) are all the possible upwind-biased schemes which contain midpoints.

(d) Flux splitting.

Similarly, the flux splitting scheme regarding upwind schemes in (3) in Section 2.2 should be applied for; in addition, the flux splitting of $f_{j+1 / 2}$ in WENOIU5-1MP can also adopt other schemes as that in WENOIU3-1MP.

(4) Variables used in WENO interpolation.

As mentioned in (4) in Section 2.2, the primitive, conservative and characteristic variables can be chosen in WENO interpolations. From literatures $[17,36]$, the last type was supposed to yield results with comparatively less oscillations usually. The key issue in the use of characteristic variables is to define proper flux, afterwards the left and right eigenvectors of its Jacobian can be acquired accordingly. Using the left eigenvectors, characteristic variables can be obtained by projecting conservative vectors from component space into characteristic field. After interpolations by characteristic variables, the results will be projected back by using the right eigenvectors. On consideration of FSP, the following procedure is chosen to construct aforementioned flux at $x_{j+1 / 2}$ as: the variables and metrics are acquired separately at $x_{j+1 / 2}$ as explained next, afterwards they are combined to form the flux required. Take the discretization of $\partial \hat{E} / \partial \xi$ in Eq. (2.2) as an example. Aforementioned flux to generate eigenvectors at $j+1 / 2$ would be $\hat{\zeta}_{x, j+1 / 2}^{\prime} E\left(Q_{j+1 / 2}^{\prime}\right)+\hat{\zeta}_{y, j+1 / 2}^{\prime} F\left(Q_{j+1 / 2}^{\prime}\right)+\hat{\zeta}_{z, j+1 / 2}^{\prime} G\left(Q_{j+1 / 2}^{\prime}\right)$, where $Q_{j+1 / 2}^{\prime}=Q_{j}$ for $h_{j+1 / 2}^{+}$ and $Q_{j+1 / 2}^{\prime}=Q_{j+1}$ for $h_{j+1 / 2}^{-}$, and where $\hat{\xi}_{x, j+1 / 2}^{\prime}=\left(\hat{\xi}_{x, j}+\hat{\xi}_{x, j+1}\right) / 2$ for simplicity. Practices show that such implementation will yield more smooth results when solving problems with shock waves, in the meanwhile FSP will not be affected. With regard to the variables to be projected $[17,36]$, both primitive and conservative variables can be used, and the latter is chosen in this study. 
Thus far, the constructions of WENOIU3 and WENOIU5 are accomplished. For completeness, the construction of the seventh-order WENOIU schemes are given in the Appendix A.

(5) Short summary on numerical implementations.

For clarity, a summary is given on numerical implementations of proposed schemes:

(a) Given upwind-biased scheme WENOIU3 (or WENOIU5), corresponding central scheme $\delta^{c,(1)}$ is derived by Eqs. (2.16) and (2.18) (or (2.28) and (2.30)). The function of $\delta^{c,(1)}$ is to compute grid metrics as following.

(b) Evaluate grid metrics such as $\hat{\xi}_{x_{i}}$ on nodes and midpoints. Firstly, metrics on nodes are acquired by Eq. (2.3) or (2.4) and $\delta^{c,(1)}$ for discretization. Next, grid metrics on midpoints are obtained by using directionally consistent interpolation, or Eq. (2.9) here.

(c) After the acquisition of grid metrics, the Jacobian $J^{-1}$ is computed by Eq. (2.5) or (2.6), and the latter is employed in this study.

(d) Evaluate flux derivatives in Eq. (2.2) by using WENOIU3 or WENOIU5.

Firstly, the splitting fluxes on nodes are computed by the method described in (3) in Section 2.2.

Next, the variables are used to interpolate the function values at midpoints by using Eqs. (2.19)-(2.24) for WENOIU3, or Eqs. (2.20)-(2.22) and (2.31)-(2.32) for WENOIU5. The variables can be of the primitive, conservative and characteristic type, and their details are referred to (4) in Section 2.3.

Afterwards, the grid metrics and flow variables on midpoints which are acquired in "(c)" are integrated to form the splitting flux, where aforementioned flux splitting scheme is employed for simplicity. As discussed in Section 2.3, for $\hat{f}_{j+1 / 2}^{ \pm}$in WENOIU3- and WENOIU5-1MP, its flux splitting scheme can adopt other methods than Lax-Friedrichslike ones.

At last, WENOIU3 or WENOIU5 are implemented to evaluate the flux derivatives.

\subsection{Discussions on proposed schemes, alternative formulation of WENO [42, 43] and hybrid WCNS $[18,44]$}

In [42], an alternative formulation of WENO (abbreviated as AF-WENO) was proposed by taking advantage of Eq. (2.12) and WENO interpolation. Later, further efforts were put to make AF-WENO achieve FSP [43]. One would expect the discussion on the relevance between current schemes and AF-WENO. In the meanwhile, it is well known that center-typed WCNS schemes [17] which are built on midpoints already have WENO-like interpolation employed and FSP achieved [24]. Furthermore, robust explicit formulation of WCNS in [44] and subsequent hybrid WCNS schemes in [18] were developed, where 
variables on midpoints and nodes were both employed. Hence, it would be valuable to extend the discussion to above schemes. For convenience, variants of WCNS in $[18,44]$ with both midpoints and nodes included are referred as H-WCNS where " $\mathrm{H}$ " denotes "hybrid".

Because of the relevance with the discussion, the following understandings are worth mentioning:

(1) The linear conservative scheme is convertible to its overall counterpart and vice visa. For example, the fourth-order center scheme of $\left(\frac{\partial f}{\partial x}\right)_{j}$ can be either $\left(\hat{f}_{j+1 / 2}-\hat{f}_{j-1 / 2}\right) / \Delta x$ with

$$
\hat{f}_{j+1 / 2}=\frac{1}{12 \Delta x}\left(-f_{j+2}+7 f_{j+1}+7 f_{j}+f_{j-1}\right)
$$

or

$$
\frac{1}{12 \Delta x}\left(-f_{j+2}+8 f_{j+1}-8 f_{j-1}+f_{j-2}\right) .
$$

(2) When the flux $f$ is split as $f=f^{+}+f^{-}$, different but related upwind schemes should be casted for $\left(\frac{\partial f^{ \pm}}{\partial x}\right)_{j}$ in order to ensure stability. For example, the third-order scheme for $\left(\frac{\partial f^{+}}{\partial x}\right)_{j}$ would be

$$
\frac{1}{6 \Delta x}\left(2 f_{j+1}^{+}+3 f_{j}^{+}-6 f_{j-1}^{+}+f_{j-2}^{+}\right)
$$

while that for $\left(\frac{\partial f^{-}}{\partial x}\right)_{j}$ would be

$$
\frac{-1}{6 \Delta x}\left(2 f_{j-1}^{-}+3 f_{j}^{-}-6 f_{j+1}^{-}+f_{j+2}^{-}\right) .
$$

(3) In construction of difference scheme, not only variables on nodes but also that on midpoints can be used for discretization. For example, in Eq. (2.17), variables on $j+1 / 2$, $j-1 / 2$ and $j$ are used to discretize $\left(\frac{\partial^{2} f}{\partial x^{2}}\right)_{j+1 / 2}$.

Still use Eq. (2.10) for discussion. Making use of Eq. (2.12) but taking the truncation order as 6, the sixth-order formulation of AF-WENO in [42] is illustrated as:

$$
\begin{aligned}
\hat{f}_{j+1 / 2}=f_{j+1 / 2}-\frac{1}{24} \Delta x^{2}\left(f^{\prime \prime}(u) u_{x}{ }^{2}+f^{\prime}(u) u_{x x}\right)_{j+1 / 2}-\frac{7}{5760} \Delta x^{4} \\
\times\left(f^{(4)}(u) u_{x}{ }^{4}+6 f^{(3)}(u) u_{x}{ }^{2} u_{x x}+4 f^{(2)}(u) u_{x} u_{x x x}\right. \\
\left.+3 f^{(2)}(u) u_{x x x}{ }^{2}+f^{\prime}(u) u_{x x x x}\right)_{j+1 / 2^{\prime}}
\end{aligned}
$$

where $f_{j+1 / 2}$ is evaluated by two-argument flux function $h_{j+1 / 2}=h\left(u_{j+1 / 2}^{L}, u_{j+1 / 2}^{R}\right)$, e.g., $h_{j+1 / 2}=f_{j+1 / 2}^{+}\left(u_{j+1 / 2}^{L}\right)+f_{j+1 / 2}^{-}\left(u_{j+1 / 2}^{R}\right)$ and etc. [43], $u_{j+1 / 2}^{L, R}$ are derived by WENO interpolation, and $f^{(n)}(u)$ denotes the Jacobian $\partial^{n} f / \partial u^{n}$. In [42], $\partial^{k} u / \partial x^{k}$ are acquired by interpolation on points $\left\{x_{j-2}, \cdots, x_{j+3}\right\}$. Hence the final formulation will be accomplished 
only when the interpolation is fulfilled, and it has the order as the minimum of that of interpolation and 6. If Eq. (2.33) is applied for curvilinear coordinates as described in Section 2.1, it is conceivable that $\mathrm{u}$ should be replaced by $\hat{Q}, f$ should correspond to transformed flux $\hat{F}$ with grid metrics included, and $\Delta x$ be replaced by grid interval of curvilinear coordinates such as $\Delta \xi$.

From the above, $\partial^{k} u / \partial x^{k}$ in AF-WENO is supposed to be approximated by center schemes in [42]. Hence its implementation is different from the upwind-biased manner of WENOIU, not to mention the employment of midpoints in the latter (see Eqs. (2.14), (2.17), (2.26) and (2.29)). Besides, in AF-WENO, combinations of high-order Jacobians $\partial^{n} f / \partial u^{n}$ and powers of $\partial^{k} u / \partial x^{k}$ are suspected to increase complexity in computation. The former will get even worse when viscosity and source terms are included. Although the authors in [42] mentioned the possibility of one-point upwind-biased scheme to approximate $\partial^{k} u / \partial x^{k}$, whether flux split is considered is not known and no more details are available. According to former understandings, the approximation of $\partial^{k} \hat{Q} / \partial x^{k}$ in curvilinear coordinate system, the counterpart of $\partial^{k} u / \partial x^{k}$, might either yield numerical instability in the use of same formula, or it might fail to achieve FSP if flux split takes place due to the metrics in $\hat{Q}$. In fact, one can find that there is neither analysis on FSP achievement nor necessary numerical validation in [42].

In [43], a new revision of AF-WENO was proposed with FSP achieved under curvilinear coordinates, where careful analysis and numerical validations were provided. In the reference, $\left(\frac{\partial^{2 l} f}{\partial \xi^{2 l}}\right)_{j+1 / 2}$ with $l=1, \cdots$, was directly discretized by center schemes rather than its expansion as functions of Jacobians and $\partial^{k} \hat{Q} / \partial \xi^{k}$. For instance, still consider Eq. (2.12) with the truncation order of 6 . In [43], the new sixth-order formulation is derived by discretizing $\left(\frac{\partial^{2} f}{\partial \xi^{2}}\right)_{j+1 / 2}$ and $\left(\frac{\partial^{4} f}{\partial \xi^{4}}\right)_{j+1 / 2}$ on $\left\{x_{j-2}, \cdots, x_{j+3}\right\}$ as:

$$
\begin{aligned}
& \left(\frac{\partial^{2} f}{\partial \xi^{2}}\right)_{j+1 / 2} \approx \frac{1}{48 \Delta \xi^{2}}\left(-5 f_{j+3}+39 f_{j+2}-34 f_{j+1}-34 f_{j}+39 f_{j-1}-5 f_{j-2}\right), \\
& \left(\frac{\partial^{4} f}{\partial \xi^{4}}\right)_{j+1 / 2} \approx \frac{1}{2 \Delta \xi^{4}}\left(f_{j+3}-3 f_{j+2}+2 f_{j+1}+2 f_{j}-3 f_{j-1}+f_{j-2}\right) .
\end{aligned}
$$

Substituting the two above into Eq. (2.12), the final sixth-order AF-WENO is acquired as

$$
\hat{f}_{j+1 / 2}=f_{j+1 / 2}+\frac{1}{3840}\left(19 f_{j+3}-137 f_{j+2}+118 f_{j+1}+118 f_{j}-137 f_{j-1}+19 f_{j-2}\right) .
$$

In the reference, the authors re-cited the mention in [42] about possibility of one-point upwind-biased approximation but with no other information. As described in Section 2.2, the achievement of FSP by upwind scheme with the presence of flux splitting needs careful analysis and specific treatments, and such studies are not referred in [43].

One can see that the main difference of WENOIU schemes from Eq. (2.34) is the upwind nature of the former. Considering the linear way of approximation of $\left(\frac{\partial^{2 k} \hat{F}}{\partial \xi^{2 k}}\right)_{j+1 / 2^{\prime}}$ the upwind recipe is supposed to favor the computational stability. 
As previously mentioned, [44] and [18] have also proposed sixth-order WCNS schemes in overall form by employing midpoints and nodes. According to previous understanding, it is easy to re-formulate them in conservative form respectively as:

$$
\begin{aligned}
& \hat{f}_{j+1 / 2}=\frac{23}{15} f_{j+1 / 2}+\frac{1}{30}\left(f_{j+3 / 2}+f_{j-1 / 2}\right)-\frac{3}{10}\left(f_{j+1}+f_{j}\right)(\text { see }[44]), \\
& \hat{f}_{j+1 / 2}=\frac{64}{45} f_{j+1 / 2}+\frac{1}{180}\left(f_{j+2}-39 f_{j+1}-39 f_{j}-f_{j-1}\right)(\text { see }[18]) .
\end{aligned}
$$

It can be seen that Eqs. (2.34)-(2.35b) are quite similar to each other, especially in case of Eqs. (2.34) and (2.35b). Hence further analysis is preferred.

Under the framework of Eq. (2.12), heuristic analysis shows that: In case of the formulation with the order $r=2 n$, the discretization of $\left(\frac{\partial^{2 k} f}{\partial x^{2 k}}\right)_{j+1 / 2}$ should take the order $r=2(n-k)$. Taking aforementioned sixth-order AF-WENO as an example, the approximation of $\left(\frac{\partial^{2} f}{\partial x^{2}}\right)_{j+1 / 2}$ should take fourth-order and that of $\left(\frac{\partial^{4} f}{\partial x^{4}}\right)_{j+1 / 2}$ should take secondorder. If the derivatives are approximated by variables on nodes, the total number of points should be $r+1$ considering the inclusion of $x_{j+1 / 2}$, while the accuracy requirement from Eq. (2.12) only offers $r$ conditions. Hence one free parameter would exist in the formulation. Realizing this fact, the general formulation with the order $r=2 n$ can be proposed as:

$$
\hat{f}_{j+1 / 2}=\alpha f_{j+1 / 2}+\sum_{l=1}^{n} a_{l}\left(f_{j+l}+f_{j-l+1}\right),
$$

where $\alpha$ serves as the free parameter. In case of $r=6$, coefficients are derived as:

$$
a_{1}=-\frac{75}{128} \alpha+\frac{37}{60}, \quad a_{2}=\frac{25}{256} \alpha-\frac{2}{15} \quad \text { and } \quad a_{3}=-\frac{3}{256} \alpha+\frac{1}{60}
$$

and when $\alpha=1$, Eq. (2.34) is recovered.

In Section 2.3, variables on midpoints are manifested to discretize $\left(\frac{\partial^{2 k} f}{\partial x^{2 k}}\right)_{j+1 / 2}$. Therefore, if $f_{j+1 / 2}$ be employed in the discretization through $\alpha \neq 1$ in Eq. (2.36), less grid points would be available, and through which H-WCNS in [18] can be acquired accordingly. Specifically, in case of $r=6$ and taking $\alpha=\frac{64}{45}$, Eq. (2.35b) can be obtained from Eq. (2.36).

More generally, more variables on midpoints can be employed to approximate $\hat{f}_{j+1 / 2}$ together with that on nodes in centered manner. It is trivial the number of midpoints should be odd and that of nodes should be even. For convenience, the odd number is denoted as $n_{0}$ and the even number is denoted as $n_{e}$. Under the framework of Eq. (2.12) with the order $r=2 n$, if the sum of no and ne satisfies: $n_{e}+n_{o}=r+1$, then one free parameter exits. The general center formulation of conservative form can be further expressed as :

$$
\hat{f}_{j+1 / 2}=\alpha f_{j+1 / 2}+\sum_{l_{1}=1}^{\left(n_{o}-1\right) / 2} b_{l_{1}}\left(f_{j+1 / 2+l_{1}}+f_{j+1 / 2-l_{1}}\right)+\sum_{l_{2}=1}^{n_{e} / 2} a_{l_{2}}\left(f_{j-l_{2}+1}+f_{j+l_{2}}\right),
$$


Table 2: Combinations of $\left(n_{o}, n_{e}\right)$ and corresponding accuracy order $r$.

\begin{tabular}{||l|c|c|c|c||}
\hline & \multicolumn{4}{|c||}{$r$} \\
\hline & $n_{e}=2$ & $n_{e}=4$ & $n_{e}=6$ & $n_{e}=8$ \\
\hline$n_{o}=1$ & 2 & $4^{\dagger, *}$ & $6^{*}$ & $8^{*}$ \\
\hline$n_{o}=3$ & $4^{\dagger}$ & 6 & 8 & 10 \\
\hline$n_{o}=5$ & 6 & $8^{\dagger}$ & 10 & 12 \\
\hline$n_{o}=7$ & 8 & 10 & $12^{\dagger}$ & 14 \\
\hline
\end{tabular}

where $\alpha$ is the free parameter. For completeness, $a_{l}$ and $b_{l}$ at the order $r$ from 4 to 8 are derived and summarized in Appendix B. In order to recover Eq. (2.35a), one can consider the case at $\left(r, n_{0}, n_{e}\right)=(6,3,4)$, where coefficients are:

$$
b_{1}=\frac{3}{10} \alpha-\frac{32}{75}, \quad a_{1}=-\frac{3}{4} \alpha+\frac{17}{20} \quad \text { and } \quad a_{2}=-\frac{1}{20} \alpha+\frac{23}{300} .
$$

One can see that when $\alpha=\frac{23}{15}$, Eq. (2.35a) is acquired. Based on above practice, possible combinations of $\left(n_{0}, n_{e}\right)$ at given $r=2 n$ can be ascertained and vice visa. For convenience, some results regarding $r$ vs $\left(n_{0}, n_{e}\right)$ are exemplified in Table 2 . In the table, the superscripts on the order indicate: $*$ means Eq. $(2.37)$ with $\left(n_{o}, n_{e}\right)$ would contain AF-WENO by taking $\alpha=1$ and H-WCNS [18] by choosing certain $\alpha$, and + means corresponding formulation would contain H-WCNS in [44] by taking appropriate $\alpha$.

The above studies indicate that given order $r=2 n$, the formulations of H-WCNS [18, 44] by Eq. (2.37) have the least grid stencil, while AF-WENO has relatively larger stencil by taking $\alpha=1$. One may wonder what function $\alpha$ can serve. According to [13,14], $\alpha$ can at least be used to optimize the dispersion relation. In order to facilitate such practice, the dispersion relation of Eq. (2.37) is derived and summarized in Appendix B.

In short, detailed discussions are made regarding proposed schemes, AF-WENO [42, $43]$ and H-WCNS $[18,44]$. Furthermore, a general center formulation in conservative form is acquired with the inclusion of midpoints and nodes, in which AF-WENO and $\mathrm{H}-\mathrm{WCNS}$ are included. Besides, schemes rather than the latter two can be naturally supplemented as shown in Appendix B. Along with the results in Section 2.3, the complete upwind-biased and center formulations are achieved thereby with co-existence of midpoints and nodes.

\section{Spectral features of WENOIU schemes}

Prior to numerical explorations of proposed schemes, their mathematical characteristics are investigated first. Two analyses are carried out, i.e., the standard Fourier analysis and that of Approximate Dispersion Relation (ADR) [40]. 


\subsection{Fourier analysis}

In [38], Fourier transformation was employed to show the spectral characteristics of a difference scheme. Consider the following generalized form of scheme:

$$
\left(\frac{\partial f(x)}{\partial x}\right)_{j} \approx \frac{1}{\Delta x}\left(\sum_{l_{1}=-N_{1}}^{M_{1}} a_{l_{1}} f_{j+l_{1}}+\sum_{l_{2}=-N_{2}}^{M_{2}} b_{l_{2}} f_{j+1 / 2+l_{2}}\right) .
$$

When $x_{j}$ is thought as the continuous variable $x$, Fourier transformation can be applied through

$$
\tilde{f}(k)=\frac{1}{2 \pi} \int_{-\infty}^{\infty} f(x) e^{-i k x} d x,
$$

where $k$ is the wave number. Usually, the scaled wave number $\kappa=k \Delta x$ is used in analysis rather than the original $k$, and a modified $\kappa^{\prime}$ with regard to Eq. (3.1) can be derived as [38]

$$
\kappa^{\prime}=-i\left(\sum_{l_{1}=-N_{1}}^{M_{1}} a_{l_{1}} e^{i l_{1} \kappa}+\sum_{l_{2}=-N_{2}}^{M_{2}} b_{l_{2}} e^{i\left(l_{2}+1 / 2\right) \kappa}\right) .
$$

By Eq. (3.2), the real and imaginary parts of $\kappa^{\prime}$ of above schemes can be obtained and are summarized in Table 3. Because the interpolation algorithms are relatively independent from the schemes, they are not incorporated into spectral relations therein. According to [38], the real part of $\kappa^{\prime}$ indicates the dispersion relation of scheme, while the imaginary part reflects the dissipation characteristics. For comparison, two additional central schemes (CS) based on midpoints by [14] are also considered for reference, i.e., the fourthorder scheme

$$
f^{\prime}{ }_{j}=\frac{1}{\Delta x}\left[\frac{9}{8}\left(f_{j+1 / 2}-f_{j-1 / 2}\right)-\frac{1}{24}\left(f_{j+3 / 2}-f_{j-3 / 2}\right)\right]
$$

and the sixth-order scheme

$$
f^{\prime}{ }_{j}=\frac{1}{\Delta x}\left[\frac{75}{64}\left(f_{j+1 / 2}-f_{j-1 / 2}\right)-\frac{25}{384}\left(f_{j+3 / 2}-f_{j-3 / 2}\right)+\frac{3}{640}\left(f_{j+5 / 2}-f_{j-5 / 2}\right)\right] .
$$

Based on the formulas in Table 3, the spectral characteristics of discussed schemes in linear forms are shown in Fig. 1. The schemes are classified into two groups according to the accuracy order, i.e., WENOIU3-1MP at $\alpha=1$, WENOIU3-2MP and CS4 as the first group in Fig. 1(a), and WENOIU5-1MP at $\alpha=1$, WENOIU5-2MP and CS6 as the second group in Fig. 1(b). In each group, results of schemes only and schemes with linear interpolations incorporated (denoted by the tag "-intp") are shown. In the first group in Fig. 1(a), when difference schemes only are concerned, CS4 shows the best performance in dispersion relation while WENOIU3-2M behaves the most dissipative. When linear interpolations are incorporated, the relative relationship of spectrum characteristics preserves within WENOIU3 schemes, and the reference CS4 shows the least dissipative and reduced performance on dispersion. In the second group in Fig. 1(b), when difference schemes only 


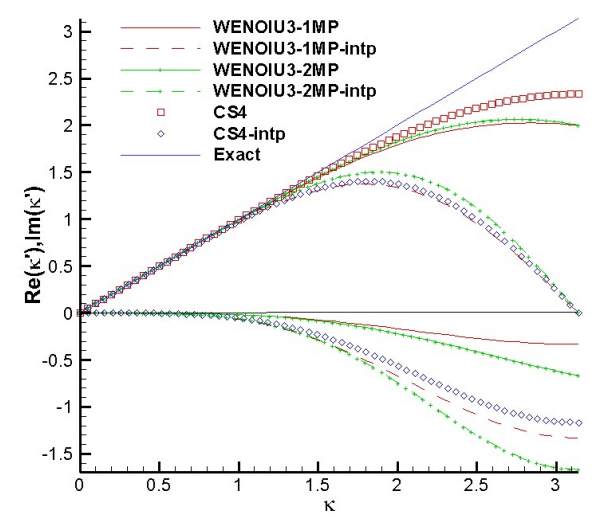

(a) WENOIU3-1MP, -2MP, CS4 and CS6

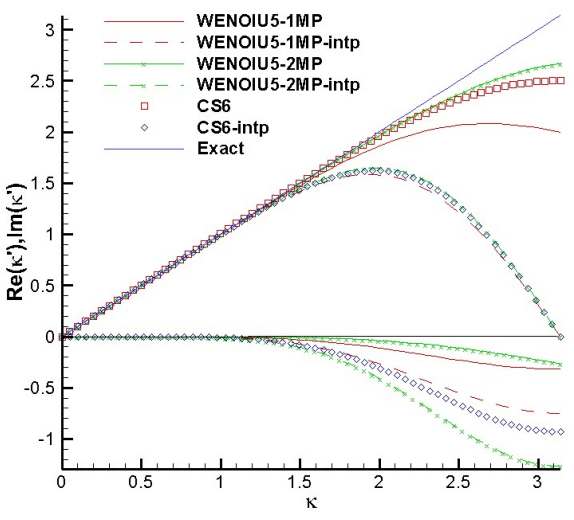

(b) WENOIU5-1MP, -2MP, CS4 and CS6

Figure 1: Dispersion and dissipation relations of difference schemes by Fourier transformation, where the suffix "-intp" indicates the scheme is incorporated with linear interpolation.

are concerned, WENOIU5-2MP and CS6 show better dispersion performance than that of WENOIU5-1MP. When linear interpolations are incorporated, all schemes show similar dispersion performance. Regarding the dissipation, CS6 indicates a dissipation larger than that of WENOIU5-1MP and WENOIU5-2MP behaves the most dissipative. The relationship of spectrum distributions among schemes with interpolation incorporated appears different from those in absence of interpolations. Such interpolation-caused phenomenon might need further analysis. Overall, the proposed schemes indicate certain amount of dissipation which is favorable for numerical stability.

Table 3: Dispersion and dissipation relations of difference schemes.

\begin{tabular}{||c|c|l||}
\hline Scheme & \multicolumn{2}{|c||}{ Spectral relation } \\
\hline \multirow{2}{*}{ WENOIU3-1MP } & Dispersion & $2 \alpha \sin \left(\frac{1}{2} \kappa\right)+\left(\frac{4}{3}-\frac{5}{4} \alpha\right) \sin (\kappa)+\left(-\frac{1}{6}+\frac{1}{8} \alpha\right) \sin (2 \kappa)$ \\
\cline { 2 - 3 } & Dissipation & $\left(-\frac{1}{3}+\frac{1}{4} \alpha\right) \cos ^{2}(\kappa)+\left(\frac{2}{3}-\frac{1}{2} \alpha\right) \cos (\kappa)+\left(-\frac{1}{3}+\frac{1}{4} \alpha\right)$ \\
\hline \multirow{2}{*}{ WENOIU3-2MP } & Dispersion & $\frac{11}{6} \sin \left(\frac{1}{2} \kappa\right)-\frac{1}{6} \sin \left(\frac{3}{2} \kappa\right)+\frac{1}{3} \sin (\kappa)$ \\
\cline { 2 - 3 } & Dissipation & $\frac{2}{3} \sin \left(\frac{1}{2} \kappa\right)^{2}\left(\cos \left(\frac{1}{2} \kappa\right)-1\right)$ \\
\hline \multirow{3}{*}{ WENOIU5-1MP } & Dispersion & $\begin{array}{l}2 \alpha \sin \left(\frac{1}{2} \kappa\right)+\left(\frac{3}{2}-\frac{175}{128} \alpha\right) \sin (\kappa) \\
+\left(-\frac{3}{10}+\frac{7}{32} \alpha\right) \sin (2 \kappa)+\left(\frac{1}{30}-\frac{3}{128} \alpha\right) \sin (3 \kappa)\end{array}$ \\
\cline { 2 - 3 } & Dissipation & $\begin{array}{l}\left(\frac{2}{15}-\frac{3}{32} \alpha \cos ^{3}(\kappa)+\left(-\frac{2}{5}+\frac{9}{32} \alpha\right) \cos ^{2}(\kappa)\right. \\
+\left(\frac{2}{5}-\frac{9}{32} \alpha\right) \cos (\kappa)+\left(-\frac{2}{15}+\frac{3}{32} \alpha\right)\end{array}$ \\
\hline \multirow{2}{*}{ WENOIU5-2MP } & Dispersion & $\frac{38}{15} \sin \left(\frac{1}{2} \kappa\right)-\frac{2}{15} \sin \left(\frac{3}{2} \kappa\right)-\frac{2}{15} \sin (\kappa)+\frac{1}{30} \sin (2 \kappa)$ \\
\cline { 2 - 3 } & Dissipation & $-\frac{4}{15} \sin \left(\frac{1}{2} \kappa\right)\left(\cos \left(\frac{1}{2} \kappa\right)-1\right)^{2}$ \\
\hline CS4 & Dispersion & $\frac{9}{4} \sin \left(\frac{1}{2} \kappa\right)-\frac{1}{12} \sin \left(\frac{3}{2} \kappa\right)$ \\
\hline CS6 & Dispersion & $\frac{189}{80} \sin \left(\frac{1}{2} \kappa\right)-\frac{25}{192} \sin \left(\frac{3}{2} \kappa\right)+\frac{3}{320} \sin \left(\frac{5}{2} \kappa\right)$ \\
\hline
\end{tabular}




\subsection{ADR analysis}

It is known that the spectral properties of nonlinear schemes differ from their linear counterparts. In order to shed some light in this regard, the Approximate Dispersion Relation method (ADR) was proposed in [40]. In ADR, the spectral characteristics of schemes were numerically derived by means of solving advection problems. Specifically, the linear advection problem in Section 4.1 is usually chosen for analysis. The initial distribution at the domain $[0,1]$ with periodic boundary is:

$$
u(x, t=0)=0.01 e^{i \kappa_{n}}
$$

where $\kappa_{n}=(2 \pi n) / N$ with $n=0, \cdots, N / 2$ and $N=122$. The computation advances over a tiny period as $t=4.0 \times 10^{-6}$. In the numerical analysis, the third-order TVD-RK3 scheme [2] is chosen for temporal discretization and aforementioned PPM techniques are employed for interpolations in WENOIUs. The results of the dispersion and dissipation relations are shown in Fig. 2, which are classified into two groups similar to that in Section 3.1. In addition, the dispersion relation of CS6 is added in the first group.

From Fig. 2(a), WENOIU3 schemes show dissipations larger than that of CS4, where WENOIU3-2MP behaves the most dissipative. Regarding the dispersion, WENOIU3$1 \mathrm{MP}$ shows the similar performance as that of CS4, while WENOIU3-2MP demonstrates an enhanced one which is comparable to that of CS6.

From Fig. 2(b), the spectral distributions of WENOIU5 and CS6 show a trend which is qualitatively similar to results with interpolations incorporated in Fig. 1(b), i.e., all dispersion relations are similar to each other and WENOIU5-1MP behaves the least dissipative.

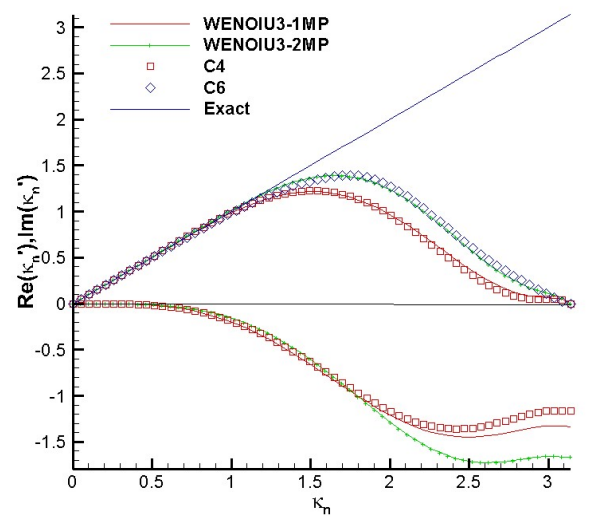

(a) WENOIU3-1MP, -2MP, CS4 and CS6

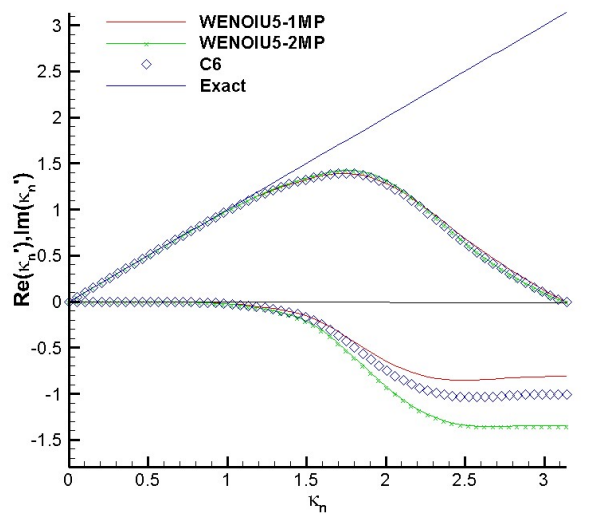

(b) WENOIU5-1MP, -2MP and CS6

Figure 2: Dispersion and dissipation relations of difference schemes by ADR. 


\section{Validations on convergence order and free-stream preservation}

\subsection{Convergence order by computations of linear advection}

In this study, the linear advection equation is chosen as

$$
u_{t}+u_{x}=0,
$$

where $x \in[-1,1]$ and uniform grids with different numbers are used. Standard fourthorder Runge-Kutta scheme is used to discretize the temporal term, and the time step is chosen with the level $(\Delta x)^{5 / 4}$ to minimize possible influence of temporal errors. The smooth initial distribution is chosen as

$$
u(x, t=0)=\sin (\pi x-\sin (\pi x) / \pi) .
$$

The computation is advanced to $t=2$. In Table 4 , numerical errors and corresponding orders are shown regarding proposed schemes, namely, WENIOU3-1MP, WENIOU3-2MP, WENIOU5-1MP, and WENIOU5-2MP. In computations, aforementioned PPM functions are employed. A special treatment is chosen for WENOIU3 schemes similar to that in [39], i.e., $I S_{0}$ and $I S_{1}$ in Eq. (2.31) on three-point stencils are employed in the same sequence instead of that in Eq. (2.23). The reason to employ new ISs comes from the fact that, on the one hand Eq. (2.23) fails to recover the third-order accuracy no matter what kinds of mapping functions are used (e.g., the one in WENO-M [5], the third- and higher order PPM functions [7]), on the other hand the linear counterpart of interpolation shown in Table 1 is testified to be able to make the order achieved. The new use of ISs can be verified easily to satisfy the accuracy requirement of weighted schemes, which manifests their qualification as indicators in the weighting procedure. By way of above manipulations, the designed orders are well established from Table 4.

Taking advantage of this case, the computation cost of WENOIU schemes is investigated to compare with that of WENO [2]. However, because in canonical WENO schemes, the issue of critical points is not concerned and various techniques subsequent are not included, WENOIU schemes in cost estimation do not employ corresponding treatments also. Concretely, smoothness indicators in WENOIU3 take the forms by

Table 4: Convergence properties of proposed schemes in linear advection problem with smooth initial condition.

\begin{tabular}{||c|cc|cc|cc|cc||}
\hline \multirow{2}{*}{$N$} & \multicolumn{2}{|c|}{ WENOIU3-1MP } & \multicolumn{2}{c|}{ WENOIU3-2MP } & \multicolumn{2}{c||}{ WENOIU5-1MP } & \multicolumn{2}{c||}{ WENOIU5-2MP } \\
\cline { 2 - 8 } & $L_{2}$ (order) & $L_{\infty}$ (order) & $L_{2}$ (order) & $L_{\infty}$ (order) & $L_{2}$ (order) & $L_{\infty}$ (order) & $L_{2}($ order) & $L_{\infty}($ order) \\
\hline 10 & $0.12(-)$ & $0.20(-)$ & $0.10(-)$ & $0.19(-)$ & $5.67 \mathrm{E}-02(-)$ & $0.11(--)$ & $5.31 \mathrm{E}-02(-)$ & $0.11(-)$ \\
20 & $2.20 \mathrm{E}-02(2.46)$ & $4.47 \mathrm{E}-02(2.18)$ & $1.75 \mathrm{E}-02(2.56)$ & $3.71 \mathrm{E}-02(2.36)$ & $2.96 \mathrm{E}-03(4.26)$ & $6.24 \mathrm{E}-03(4.16)$ & $2.58 \mathrm{E}-03(4.36)$ & $5.43-\mathrm{E} 03(4.29)$ \\
40 & $3.64 \mathrm{E}-03(2.59)$ & $7.78 \mathrm{E}-03(2.52)$ & $2.81 \mathrm{E}-03(2.64)$ & $6.09 \mathrm{E}-03(2.61)$ & $1.06 \mathrm{E}-04(4.81)$ & $2.10 \mathrm{E}-04(4.89)$ & $8.94 \mathrm{E}-05(4.84)$ & $1.73-\mathrm{E} 04(4.97)$ \\
80 & $5.01 \mathrm{E}-04(2.86)$ & $1.05 \mathrm{E}-03(2.88)$ & $3.78 \mathrm{E}-04(2.89)$ & $7.87 \mathrm{E}-04(2.95)$ & $3.22 \mathrm{E}-06(5.03)$ & $6.70 \mathrm{E}-06(4.97)$ & $2.69 \mathrm{E}-06(5.05)$ & $5.48-\mathrm{E} 06(4.98)$ \\
160 & $6.42 \mathrm{E}-05(2.96)$ & $1.28 \mathrm{E}-04(3.03)$ & $4.82 \mathrm{E}-05(2.97)$ & $9.61 \mathrm{E}-05(3.03)$ & $9.84 \mathrm{E}-08(5.03)$ & $2.10 \mathrm{E}-07(5.00)$ & $8.12 \mathrm{E}-08(5.05)$ & $1.71-\mathrm{E} 07(5.00)$ \\
320 & $8.08 \mathrm{E}-06(2.99)$ & $1.60 \mathrm{E}-05(3.00)$ & $6.06 \mathrm{E}-06(2.99)$ & $1.20 \mathrm{E}-05(3.00)$ & $3.04 \mathrm{E}-09(5.01)$ & $6.55 \mathrm{E}-09(5.00)$ & $2.49 \mathrm{E}-09(5.02)$ & $5.35-\mathrm{E} 09(5.00)$ \\
640 & $1.01 \mathrm{E}-06(3.00)$ & $2.00 \mathrm{E}-06(3.00)$ & $7.59 \mathrm{E}-07(3.00)$ & $1.50 \mathrm{E}-06(3.00)$ & $9.46 \mathrm{E}-11(5.01)$ & $2.05 \mathrm{E}-10(5.00)$ & $7.71 \mathrm{E}-11(5.02)$ & $1.67-\mathrm{E} 10(5.00)$ \\
\hline
\end{tabular}


Eq. (2.23), and mapping functions are not invoked for WENOIU schemes. The computations are carried out with the grid number 640 and run for 4000 steps. The consequences are: WENOIU3 schemes will cost $107.69 \%$ CPU time as that of WENO3, and WENOIU5 schemes will cost $134.88 \%$ CPU time as that of WENO5. Among WENOIU3 schemes, the interpolation is implemented and kept exclusively, therefore their time cost are almost the same. Similar situation happens to WENOIU5 schemes.

In the following, $I S_{0}$ and $I S_{1}$ by Eq. (2.31) are used for WENOIU3 schemes instead of Eq. (2.23) in order to achieve designed accuracy.

\subsection{Free-stream preservation on randomized grids}

In this case, an initial uniform flow condition is employed with the Mach number 0.5 and the randomized grids described in (1) of Section 5.1 are used. Using the proposed thirdand fifth-order schemes, the computation advances until $t=10$ with the time step $\Delta t=$ 0.01 . $L_{2}$ errors of velocity component $v$, which is theoretically zero, are chosen and shown in Table 5 for illustration. In the computation, flux splitting uses the aforementioned LaxFriedrichs-type scheme for simplicity. From the table, the free-stream preservation is well achieved by WENOIU schemes on randomized grids.

Table 5: $L_{2}$ errors of $v$ component in FSP test on randomized grids.

\begin{tabular}{||c|c|c|c|c||}
\hline & WENOIU3-1MP & WENOIU3-2MP & WENOIU5-1MP & WENOIU5-2MP \\
\hline$L_{2}(v)$ & $1.820 \mathrm{E}-15$ & $1.500 \mathrm{E}-15$ & $2.401 \mathrm{E}-15$ & $2.512 \mathrm{E}-15$ \\
\hline
\end{tabular}

\section{Numerical examples}

In all examples of the section, Euler equations are solved, PPM methods are employed for interpolations in WENOIU, and the third-order TVD-RK3 method [2] is chosen for temporal discretization. At first, grid generations of 2-D computations are described.

\subsection{Grid generations in 2-D problems}

(1) Grids for vortex preservation.

Two grids are employed, namely the uniform and randomized grids. The latter is generated by:

$$
\begin{aligned}
& x_{i, j}=-\frac{L}{2}+\frac{L}{I_{\max }-1}\left[(i-1)+2 A_{i, j}(\operatorname{Rand}(0,1)-0.5) \operatorname{Rand}(0 \mid 1)\right], \\
& y_{i, j}=-\frac{L}{2}+\frac{L}{J_{\max }-1}\left[(j-1)+2 A_{i, j}(\operatorname{Rand}(0,1)-0.5)(1-\operatorname{Rand}(0 \mid 1))\right],
\end{aligned}
$$

where $L=16, A_{i j}=0.45$ at $i=5, \cdots, I_{\max }-4$ or $j=5, \cdots, J_{\max }-4$ otherwise $A_{i j}=0, \operatorname{Rand}(0,1)$ is a random function ranging from 0 to 1 , and $\operatorname{Rand}(0 \mid 1)$ is one having the value 0 or 


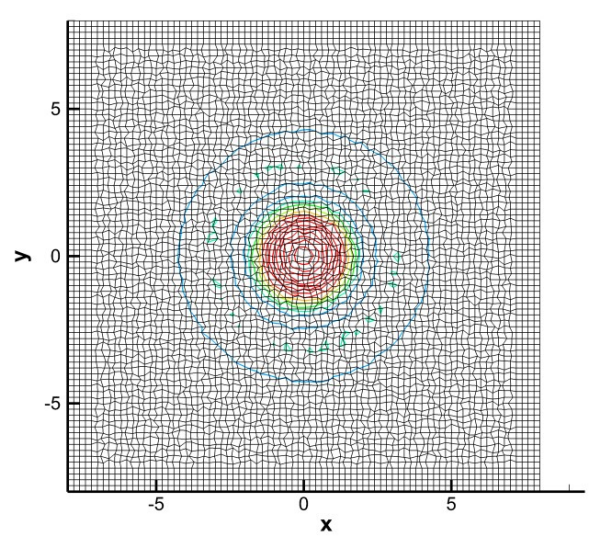

Figure 3: $81 \times 81$ Randomized grids of vortex preservation with background by initial vorticity contours.

1. In this study, $\left(I_{\max } \times J_{\max }\right)=(81 \times 81)$. The grids are shown in Fig. 3 with the initial vorticity contours as the background. It is worth mentioning that the grid randomization is so serious that further increase of $A_{i j}$ will generate negative grid-cell area. Besides being used in vortex preservation, the randomized grids are also applied in the test of free-stream preservation in Section 4.2. The uniform grids have the same dimensions and physical range as that of the randomized ones.

It should be mentioned that the use of randomized grids does not indicate their practicability in applications. The only purpose is to provide circumstance to test the capability of proposed schemes on seriously distorted grids, and to check if the schemes could achieve FSP there.

(2) Grids for supersonic flow around inviscid cylinder.

A cylinder with the diameter as one is considered. Similarly, the smooth grids and the randomized ones are generated. The grid dimension $\left(I_{\max } \times J_{\max }\right)$ is $121 \times 41$, which corresponds the streamwise and normal-wise directions. The smooth grids are generated by ordinary transfinite interpolation, while the randomized ones are acquired by randomizing the grids in a window of the former as that in (1). The indices of window is $\left(6, I_{\max }-5\right) \times\left(7, J_{\max }-6\right)$, and the randomizing factor $A_{i j}$ is 0.2 . In Fig. 4 , the half of referred window together with grids are shown with the background by pressure contours. From the figure, it can be seen that the shock wave and some flows behind are contained in the region with randomized grids.

(3) Grids for Riemann problem and shock-vortex interaction.

The physical domains are both rectangular in two cases. The one in Riemann problem is $[0,1] \times[0,1]$ with $400 \times 400$ grids, and that in shock-vortex interaction is $[-20,10] \times$ $[-15,15]$ with $600 \times 600$ grids. Two types of grids are chosen, i.e., the uniform grids and the randomized ones. For the latter, the grids are generated just as that in (1), where the 


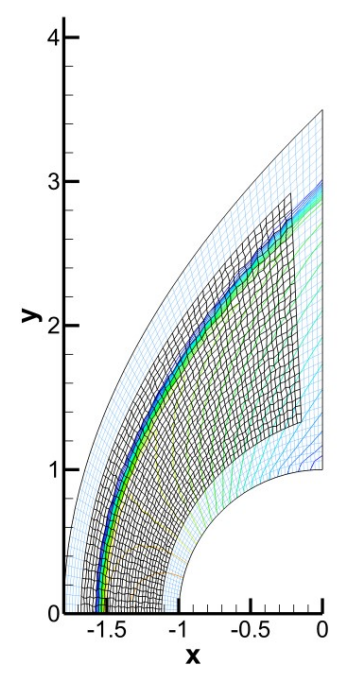

Figure 4: $121 \times 41$ grids of cylinder with background by pressure contours, where randomization is imposed in a local window of the domain.

factor $A_{i j}$ for inner randomized grids is 0.2 , and where four and nine layers of uniform grids are included in boundaries respectively in Riemann problem and shock-vortex interaction.

\subsection{One-dimensional problems}

The purpose of tests is to check the qualification of shock capturing of proposed schemes, and therefore validates the nonlinear implementation by WENO interpolation. In subsequent figures, the results of WENO3 and WENO5 are shown as well.

(1) Sod problem.

The initial distributions of the problem at the range $[-5,5]$ are

$$
(\rho, u, p)= \begin{cases}(1.000,0,1.0), & -5 \leq x<0 \\ (0.125,0,0.1), & 0 \leq x \leq 5\end{cases}
$$

and the computation is advanced to $t=2.0$ on 200 uniform grids with $\Delta t=0.01$. On checking, WENOIU3 schemes yield results quite similar to each other, and so do WENOIU5 schemes. Hence for brevity, only the density distribution of WENOIU3-1MP and WENOIU5-2MP in proposed schemes are shown as representatives in Figs. 5 and 6. From the figures, the fifth-order scheme indicates slightly better performance at the corners of contact discontinuity than that of the third-order scheme. In the meanwhile, the difference between results of WENOIU3-1MP and WENO3 is quite small, and similar situation exists between that of WENOIU5-2MP and WENO5. 


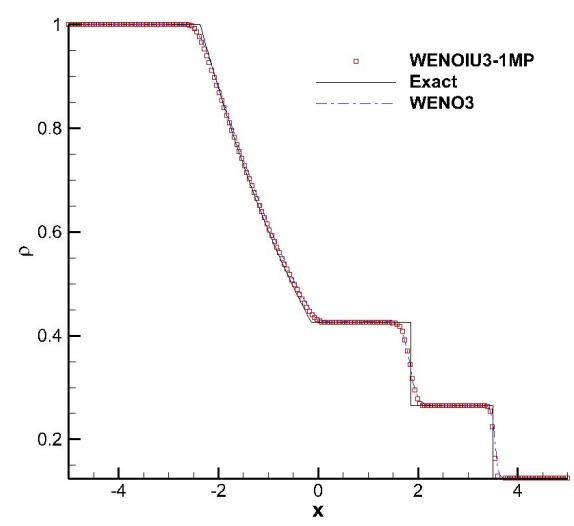

Figure 5: Density distribution of Sod problem by WENOIU3-1MP.

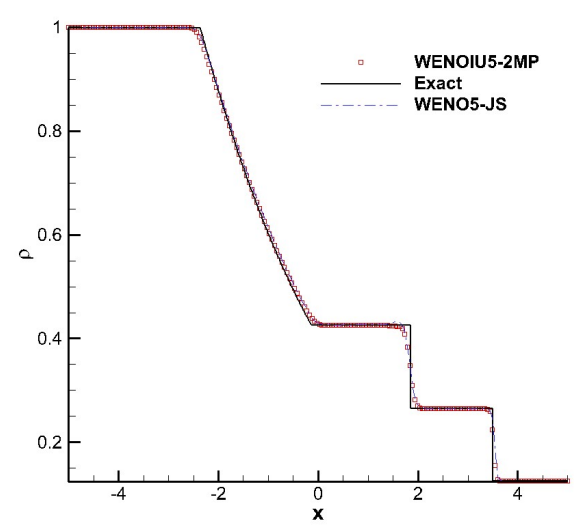

Figure 6: Density distribution of Sod problem by WENOIU5-2MP.

(2) Shu-Osher problem.

The initial distributions of the problem at the range $[-5,5]$ are

$$
(\rho, u, p)= \begin{cases}(3.857143,2.629369,10.3333) & x<-4 \\ (1+0.2 \sin (5 x), 0,1), & x \geq-4\end{cases}
$$

and the computation is advanced to $t=1.8$ on 400 uniform grids with $\Delta t=0.001$. WENOIU3 schemes show similar results to each other again and only that of WENOIU3$2 \mathrm{MP}$ is shown in Fig. 7 for brevity. One can check that better resolution is acquired by WENOIU3-2MP if compared with that of canonical WENO3, and the improvement should be attributed to the new use of IS discussed before. In Fig. 8, WENOIU5-1MP shows well resolutions on the small-scale structures after the shock if compared with 


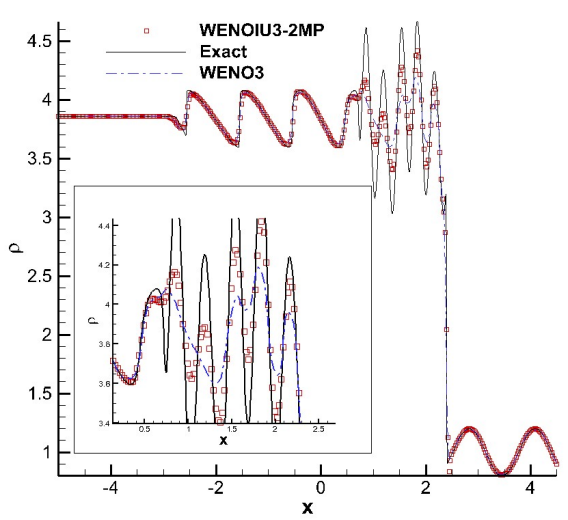

Figure 7: Density distribution of WENOIU3-2MP for Shu-Osher problem with the comparison of WENO3.

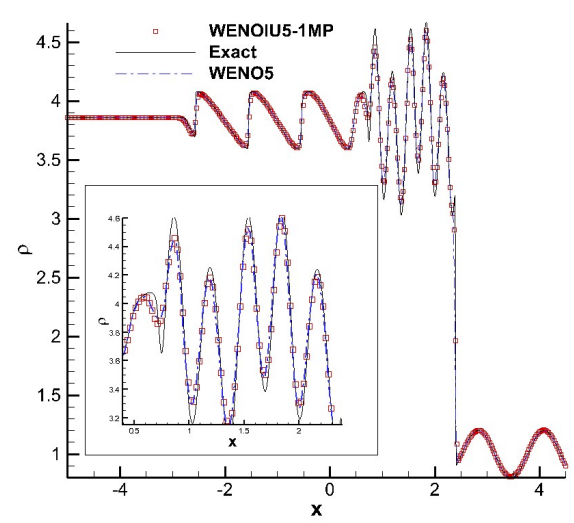

Figure 8: Density distribution of WENOIU5-1MP for Shu-Osher problem with the comparison of WENO5.

that of WENOIU3-2MP, and the result of WENOIU5-2MP is omitted for brevity again because of its similarity to that of WENOIU5-1MP. Besides, the difference between results of WENOIU5-1MP and WENO5 is quite small.

\subsection{Two-dimensional problems}

(1) Vortex preservation.

This problem is to check if a moving vortex could preserve its shape in grids introduced in Section 5.1 with periodic boundaries, especially on distorted grids by randomization. The flow is non-dimensionalized by the density and the speed of sound, and the free-stream Mach number is 1. 


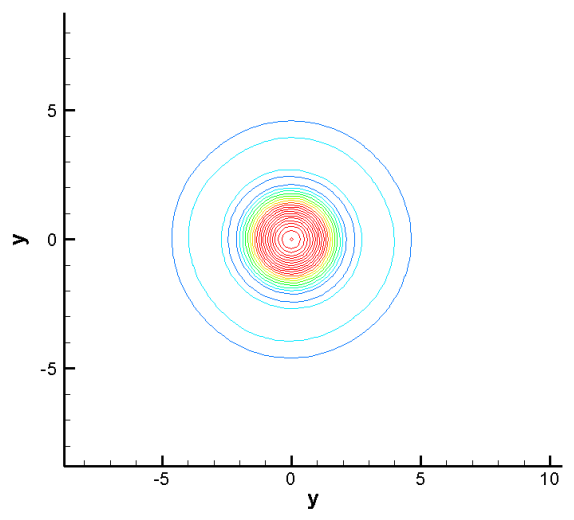

(a) Results on uniform grids

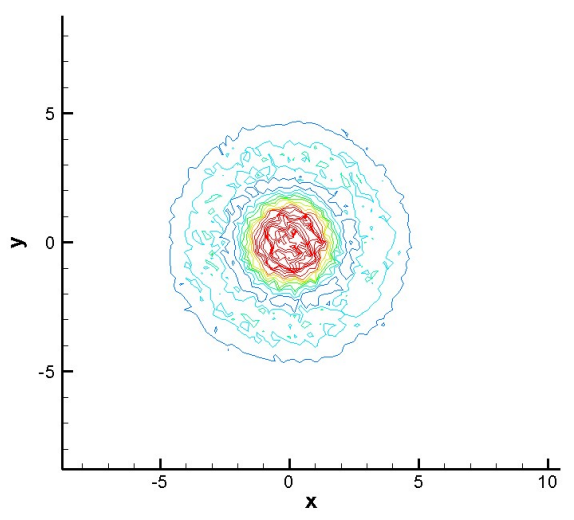

(b) Results on randomized grids

Figure 9: Vorticity contours of vortex preservation problem on two $81 \times 81$ grids by using WENOIU3-1MP (Contours from 0 to 0.7 with number 21 ).

An isentropic vortex is initially superimposed on the uniform flow at $\vec{r}_{0}=(0,0)$ as [24]

$$
\left\{\begin{array}{l}
(\delta u, \delta v)=\varepsilon \tilde{r} e^{\alpha\left(1-\tilde{r}^{2}\right)}(\sin \theta,-\cos \theta) \\
\delta T=-\frac{(\gamma-1) \varepsilon^{2}}{4 \alpha \gamma} e^{2 \alpha\left(1-\tilde{r}^{2}\right)} \\
\delta S=\delta\left(p / \rho^{\gamma}\right)=0
\end{array}\right.
$$

where $\tilde{r}=\left|\vec{r}-\vec{r}_{0}\right| / r_{c}, r_{c}=1, \alpha=0.204, \varepsilon=0.3$, and $\gamma=1.4$. The computation runs from the initial conditions at $\Delta t=0.01$ till $t=16$, which corresponds to one movement circle of vortex to return to its initial place through the periodic boundary.

WENOIU3 and WENOIU5 schemes are respectively tested in uniform and randomized grids. In the tests of WENOIU3 schemes, WENOIU3-2MP shows quite similar result to that of WENOIU3-1MP, therefore the vorticity contours of the former are omitted here. Similarly, only the contours of WENOIU5-2MP are shown for representative.

On the uniform grids, WENOIU3-1MP yields a result with quite smooth appearance and well circular shape as shown in Fig. 9(a). The quantitative comparison of WENOIU3 with the exact solution is fairly well as shown in Fig. 10(a). On randomized grids (Fig. 9(b)), WENOIU3-1MP yields a result with reasonable preservation of vortex, which is comparable to that in [24]. The preservation is also confirmed by the quantitative distributions in Fig. 10(b). It is worthwhile to mention that the vorticity contours are especially chosen for visualization rather than isobar contours, because the latter usually show smoother appearance and might conceal potential problems. As shown in Fig. 3, even the initial vorticity contours on randomized grids do not distribute smoothly enough due to the bad grid quality. 


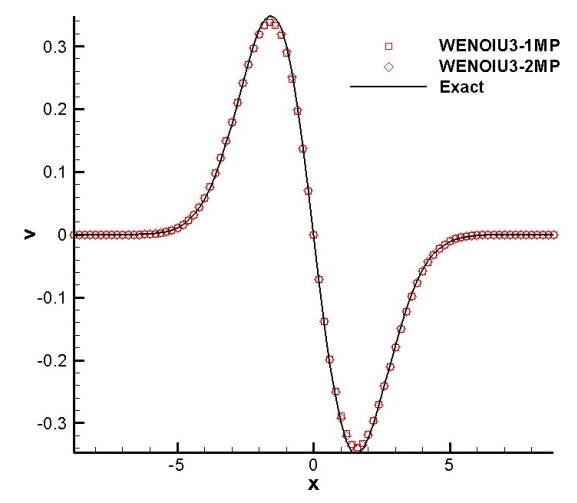

(a) Results on uniform grids

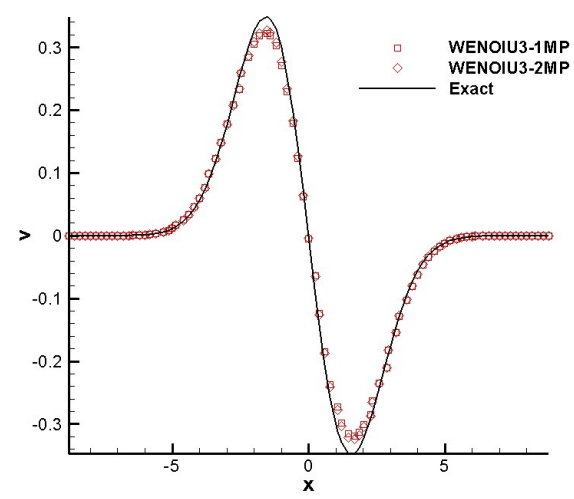

(b) Results on randomized grids

Figure 10: Distributions of $v$-component along the line at $j=\left(J_{\max } / 2\right)+1$ of vortex preservation problem on two $81 \times 81$ grids by using WENOIU3 schemes.

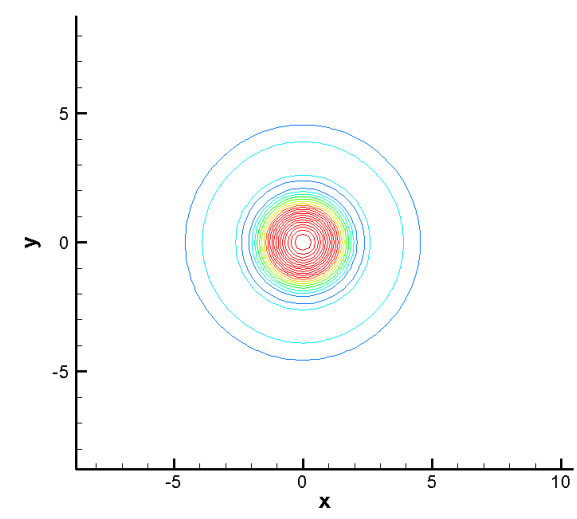

(a) Results on uniform grids

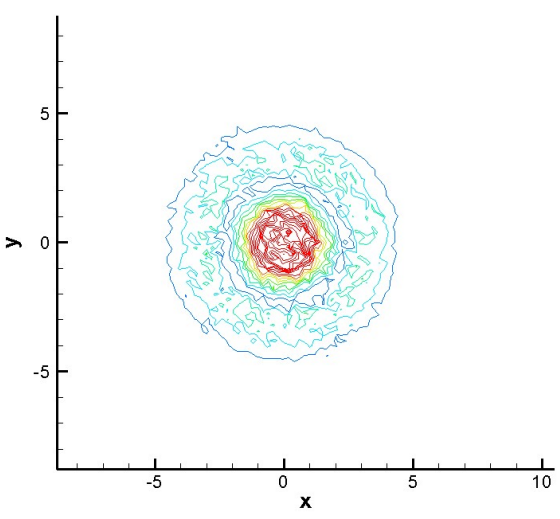

(b) Results on randomized grids

Figure 11: Vorticity contours of vortex preservation problem on two $81 \times 81$ grids by using WENOIU5-2MP (Contours from 0 to 0.7 with number 21 ).

The results of WENOIU5-2MP are shown in Figs. 11 and 12. From the figures, the structure of vortex appears qualitatively similar to that of WENOIU3-1MP. Quantitatively, WENOIU5-2MP performs less dissipatively and shows slightly better agreement with the exact distribution.

One may be interested in the performances of WENO schemes. In this regard, the results by WENO5 are shown for demonstration. Because such computations on uniform and randomized grids are already made in $[16,25,43]$, it ought to be objective and persuasive to quote corresponding results for discussion, and regarding vorticity contours 


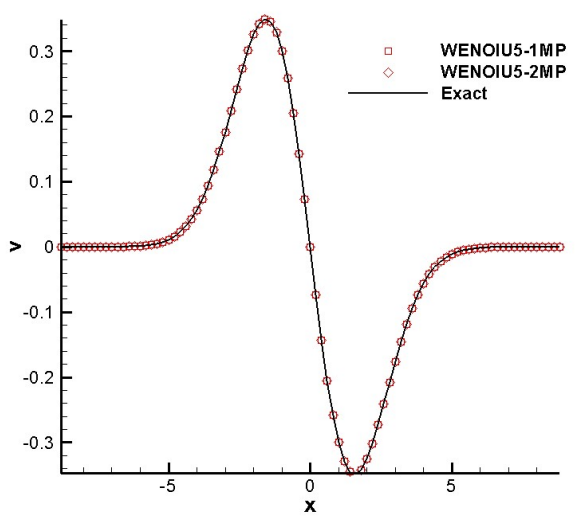

(a) Results on uniform grids

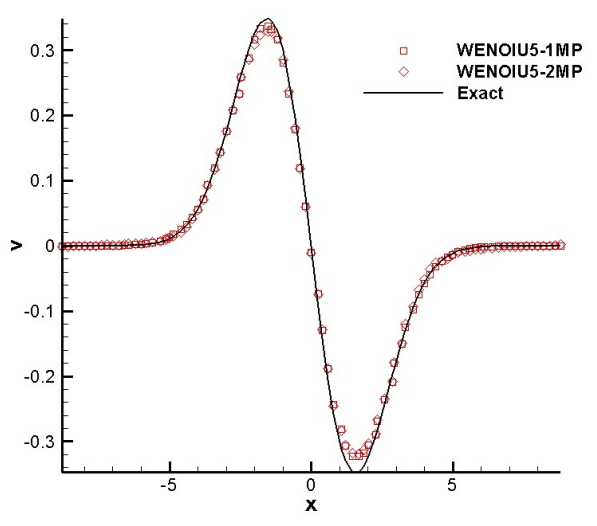

(b) Results on randomized grids

Figure 12: Distributions of $v$-component along the line at $j=\left(J_{\max } / 2\right)+1$ of vortex preservation problem on two $81 \times 81$ grids by using WENOIU5 schemes.

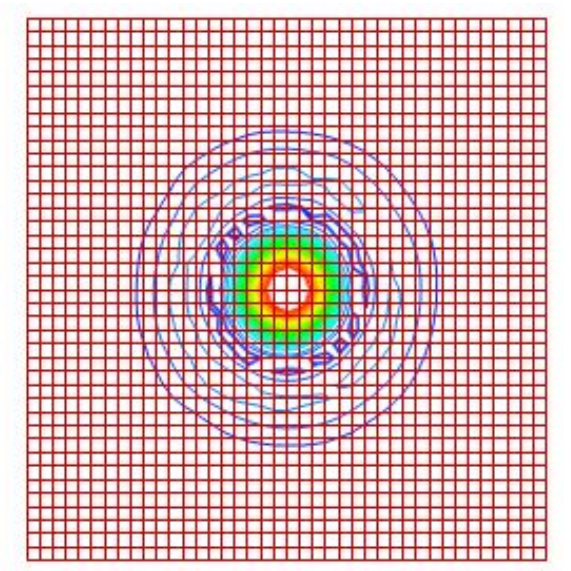

(a) Results on uniform grids with the courtesy from [43]

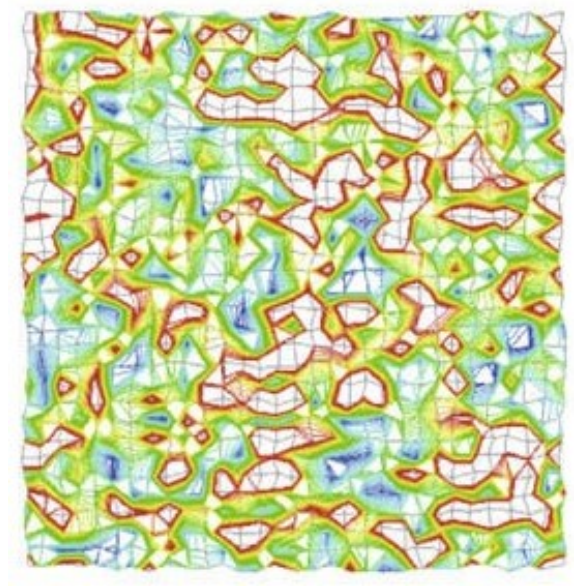

(b) Results on randomized grids with the courtesy from [16]

Figure 13: Vorticity contours of vortex preservation problem on uniform and randomized grids by WENO5 with the courtesy from $[16,43]$.

are shown in Fig. 13 with the courtesy from the references. It is apparent that although WENO5 can well perform on uniform grids, it yields vortex out of shape on randomized grids because of dissatisfaction of FSP.

(2) Supersonic inviscid flow around the cylinder at $M_{\infty}=4$.

Although this case may not be quite noticed by fundamental researches, it is concerned by engineering due to its application background in aircrafts. Despite the com- 


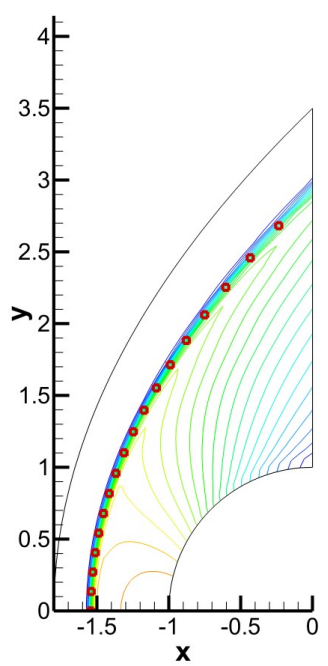

(a) Results of WENOIU3-1MP on uniform grids

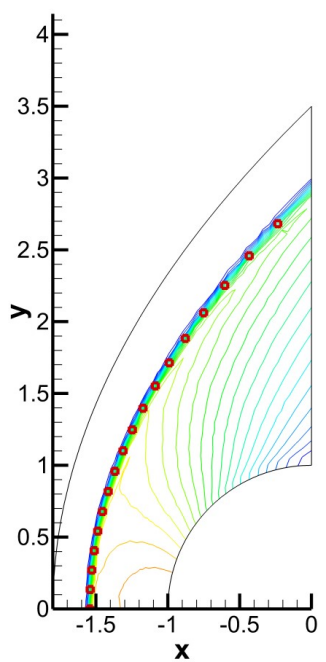

(b) Results of WENOIU3-2MP on randomized grids

Figure 14: Pressure contours of supersonic cylinder flow on two $121 \times 41$ grids by using WENOIU3 schemes (Contours from 1.2 to 5.2 with number 21; Dots: solution from Lyubinov\& Rusanov [41]).

putation fulfillment by second-order TVD schemes, simulations on randomized meshes are seldom tested by high-order schemes. The computation is carried out at $\Delta t=0.001$ and advanced till $t=400$. First, the proposed third-order WENOIU3 schemes are tested. On both grids, the schemes produce qualitatively similar results, therefore the results are selectively shown in Fig. 14. From the figure, the capability of proposed methods is manifested to resolve shock wave on grids with bad quality.

Next, WENOIU5 schemes are tested as well. In Fig. 15, similar characteristics are observed as that in computations by using WENOIU3. Qualitatively, the contours on randomized grids in Fig. 15(b) appear relatively unsmooth than that in Fig. 15(b), which might arise from the less dissipation with the increase of order.

In order to check quantitatively, the pressure distributions along body surface of WENOIU3-2MP and WENOIU5-1MP on randomized grids are displayed as representatives in Fig. 16. The results are compared with the asymptotic solution by Lyubinov and Rusanov [41]. From the figure, well agreements are obtained between the computations and the asymptotic solution.

One may also wonder how WENO would perform, and in this respect similar investigation by WENO5 are known to explore already. Concretely in [25,43], supersonic flows around cylinder by WENO5 have been simulated on smooth and randomized grids, and corresponding results are cited in Fig. 17 similarly with the courtesy from the references. The figure indicates again that although WENO5 can well resolve shocks on smooth 


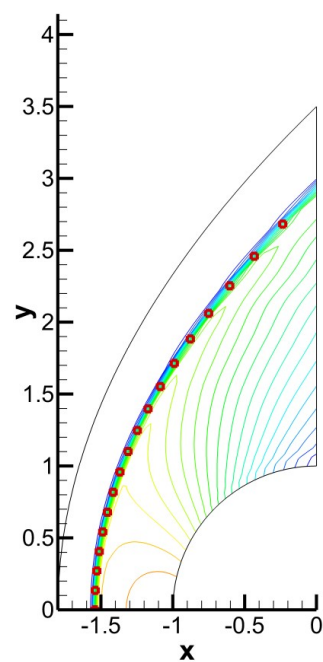

(a) Results of WENOIU5-2MP on uniform grids

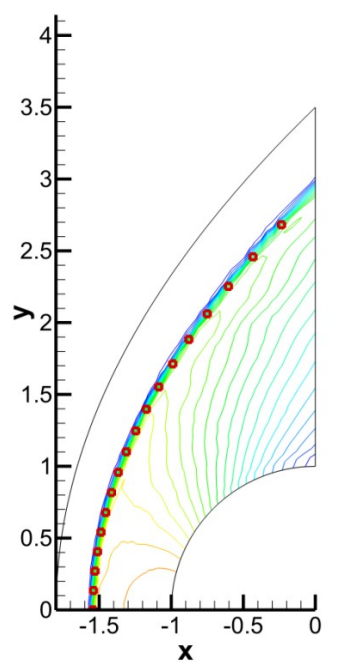

(b) Results of WENOIU5-1MP on randomized grids

Figure 15: Pressure contours of supersonic cylinder flow on two $121 \times 41$ grids by using WENOIU5 schemes (Contours from 1.2 to 5.2 with number 21; Dots: solution from Lyubinov\& Rusanov [41]).

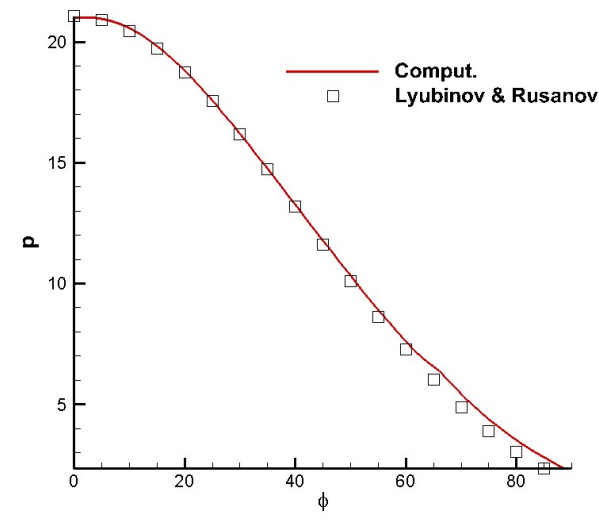

(a) Results of WENOIU3-2MP on uniform grids

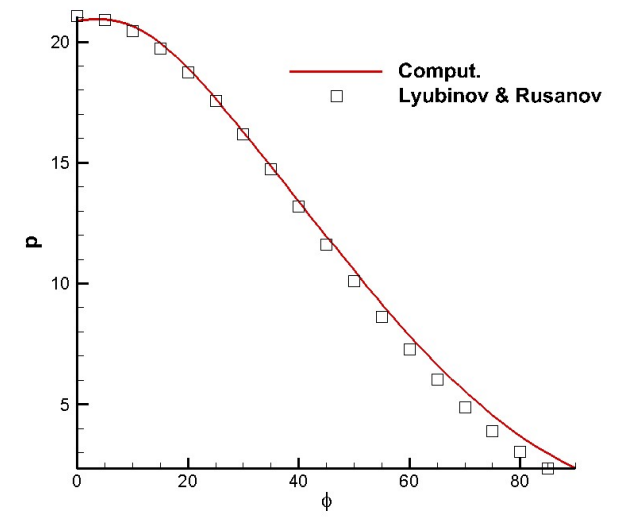

(b) Results of WENOIU5-1MP on randomized grids

Figure 16: Pressure distributions along wall surface of supersonic cylinder flow on $121 \times 41$ randomized grids by WENOIU3-2MP and WENOIU5-1MP (Dots: solution from Lyubinov and Rusanov [41]).

grids, it engenders a result with large oscillations on randomized grids with little application value, which indicates the significance of FSP achievement in computations on distorted grids. 


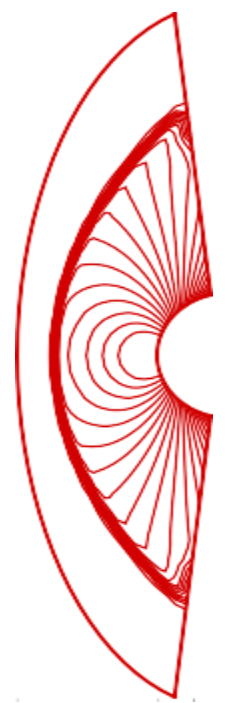

(a) Results on smooth grids with the courtesy from [43]

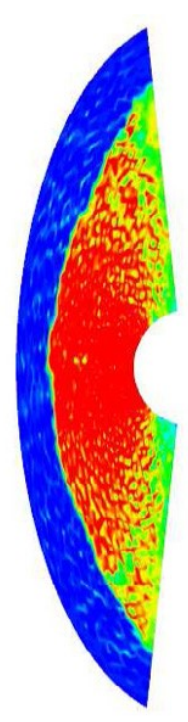

(b) Results on randomized grids with the courtesy from [25]

Figure 17: Pressure contours of supersonic cylinder flow by using WENO5 schemes from the courtesy from [25, 43].

(3) Riemann problem.

This problem illustrates the capability of proposed schemes for resolving shock wave and slip line, especially under tough circumstance where randomized grids are used. The following initial condition is used for four sub-divisions of the domain $[0,1]^{2}$ as:

$$
\begin{array}{lllll}
{[0.5,1] \times[0.5,1]:} & \rho_{1}=1, & p_{1}=1, & u_{1}=0, & v_{1}=-0.3, \\
{[0,0.5] \times[0.5,1]:} & \rho_{2}=2, & p_{2}=1, & u_{2}=0, & v_{2}=0.3, \\
{[0,0.5] \times[0,0.5]:} & \rho_{3}=1.0625, & p_{3}=0.4, & u_{3}=0, & v_{3}=0.8145, \\
{[0.5,1] \times[0,0.5]:} & \rho_{4}=0.5313, & p_{4}=0.4, & u_{4}=0, & v_{4}=0.4276 .
\end{array}
$$

The Euler equations are solved on $400 \times 400$ grids described previously, and the computations proceed to $t=0.3$. Two time steps are employed, i.e., $\Delta t=0.001$ for uniform grids and $\Delta t=0.0001$ for randomized grids. The reduction of time step in the latter is due to the decreased numerical stability. The results of WENOIU3 schemes are first discussed. On uniform grids, the result of WENOIU3-1MP is quite similar to that of WENOIU3-2MP, therefore only the latter is shown in Fig. 18(a) for representative. On randomized grids, WENOIU3-1MP fulfills the computation with the result shown in Fig. 18(b), and the fulfillment manifests its capability to resolve shock and slip line on grids with bad quality. However, WENOIU3-2MP fails to accomplish the test even if Dt is largely decreased, which implies its relatively weaker numerical stability.

Next, the results of WENOIU5 schemes are checked and shown in Fig. 19. On uniform grids, WENOIU5-1MP yields quite smooth result while WENOIU5-2MP generates 


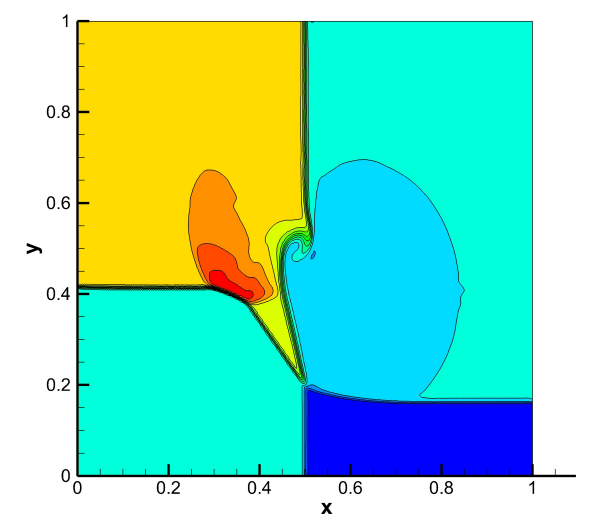

(a) Results of WENOIU3-2MP on uniform grids

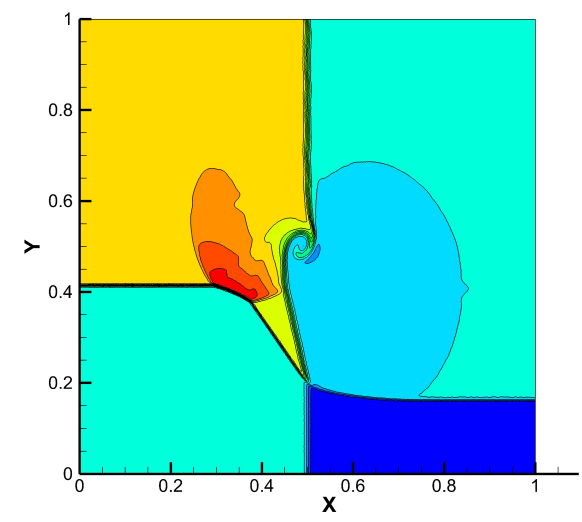

(b) Results of WENOIU3-1MP on randomized grids

Figure 18: Density contours of Riemann problem on two $401 \times 401$ grids by using WENOIU3 schemes (Contours from 0.6 to 2.3 with number 14 ).

a result with some oscillations. Such performance of WENOIU5-2MP might contribute to the generation of subtle structures at the slip line. On randomized grids, both schemes accomplish the computations and yield similar results. If compared with the results by WENOIU3 schemes, WENOIU5 schemes indicate better resolutions on the description of the slip line.

Similarly, in this case WENO schemes can accomplish satisfactory computations on smooth grids but yields unsuccessful results with oscillations on randomized grids, which indicates the importance of FSP achievement once again. For illustration, results on smooth grids are shown in Fig. 20, while results on randomized grids are of oscillations similar to Fig. 17(b) and therefore are omitted for brevity.

(4) Shock-vortex interaction.

The case describes a strong shock with $M_{s}=3$ interacting with a strong vortex, where complex flow structures are generated along with the interaction. The computational domain is: $[-20,10] \times[-15,15]$, where a shock wave is initially located at $x=0$. The upper and lower boundaries are periodic. The initial flow field after the shock can be defined by Rankine-Hugoniot relations. An isentropic vortex is initially superimposed ahead of the shock wave as:

$$
\begin{aligned}
& u_{\theta}=\Gamma\left|\vec{r}-\vec{r}_{0}\right| / r_{c} e^{\left[\left(1-\left|\vec{r}-\vec{r}_{0}\right|^{2}\right) / 2\right],} \\
& p(r)=\frac{1}{\gamma M_{\infty}^{2}}\left[1-\frac{\gamma-1}{2} \Gamma^{2} \exp \left(1-\left|\vec{r}-\vec{r}_{0}\right|^{2}\right)\right]^{\gamma /(\gamma-1)}, \\
& \rho(r)=\left[1-\frac{\gamma-1}{2} \Gamma^{2} \exp \left(1-\left|\vec{r}-\vec{r}_{0}\right|^{2}\right)\right]^{1 /(\gamma-1)},
\end{aligned}
$$




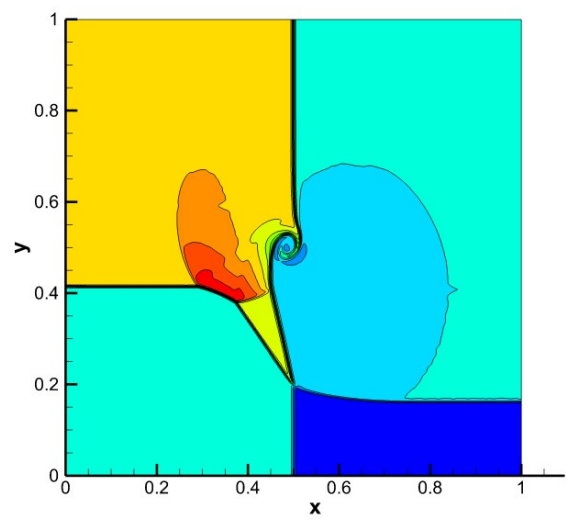

(a) Results of WENOIU5-1MP on uniform grids

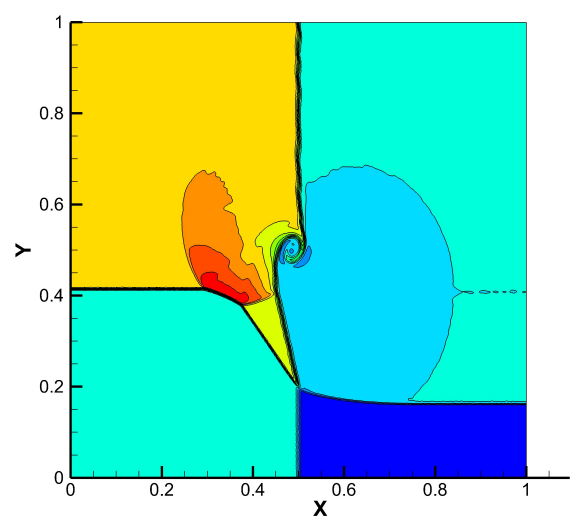

(c) Results of WENOIU5-1MP on randomized grids

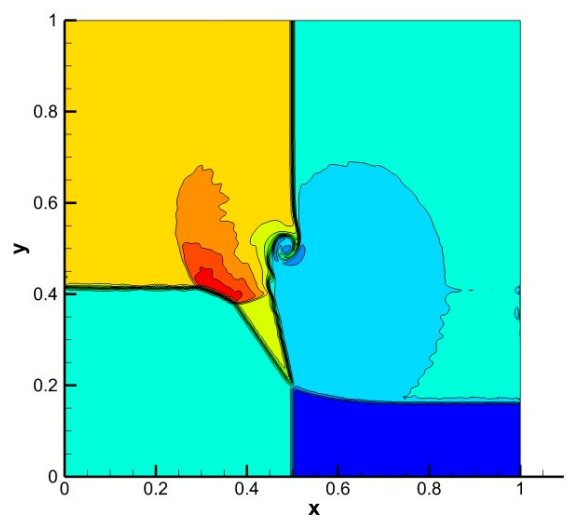

(b) Results of WENOIU5-2MP on uniform grids

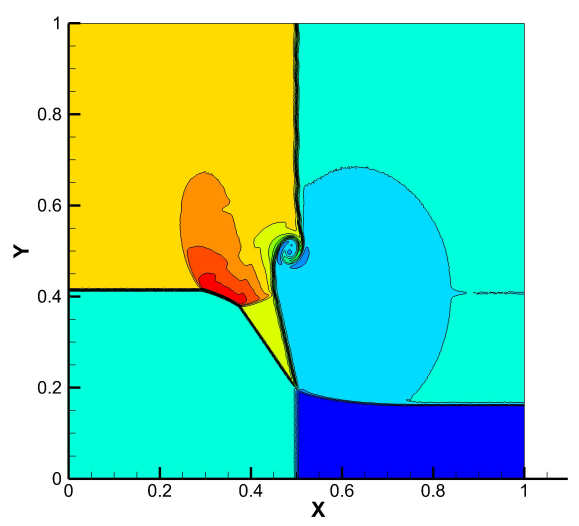

(b) Results of WENOIU5-2MP on randomized grids

Figure 19: Density contours of Riemann problem on two $401 \times 401$ grids by using WENOIU5 schemes (Contours from 0.6 to 2.3 with number 14 ).

where $u_{\theta}$ denotes the tangential velocity of the vortex, $\vec{r}_{0}=(4,0)$ represents the initial position of the vortex, and $G$ is the vortex strength with the value 0.4 . The computation runs to $t=15$ with $\Delta t=0.01$.

The results of WENOIU3 schemes are checked first. On uniform grids, both WENOIU3-1MP and -2MP schemes yield results with no qualitative difference, therefore only the density contours of WENOIU3-2MP are drawn in Fig. 21(a) for representative. On randomized grids, WENOIU3-1MP works normally with the result shown in Fig. 21(b), however WENOIU3-2MP fails even if $\Delta t$ is largely decreased. This result seems to indicate again that WENOIU3-2MP would be less stable or robust when the grids are of poor quality. 


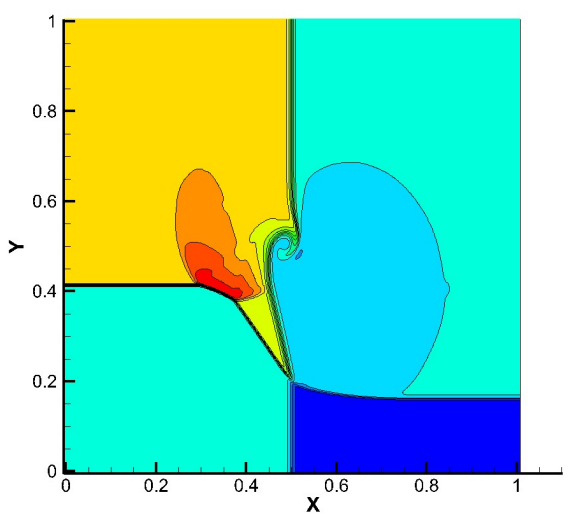

(a) Results of WENO3

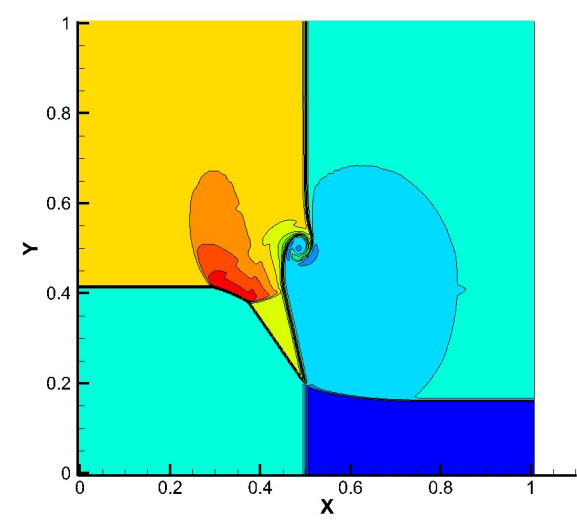

(b) Results of WENO5

Figure 20: Density contours of Riemann problem on two $401 \times 401$ grids by using WENO schemes (Contours from 0.6 to 2.3 with number 14 ).

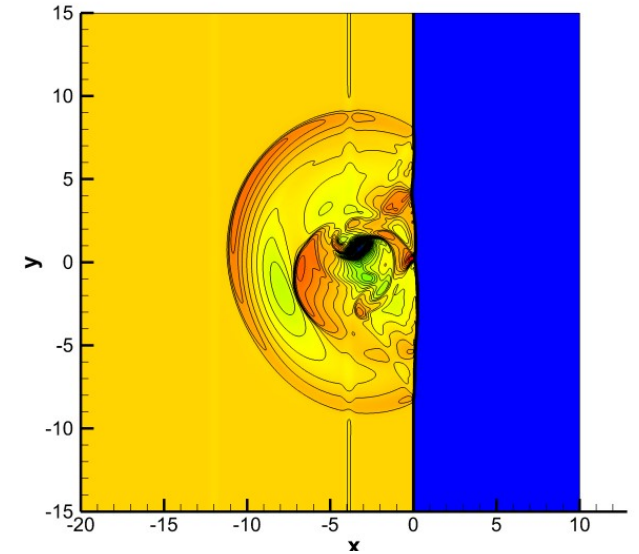

(a) Results of WENOIU3-2MP on uniform grids

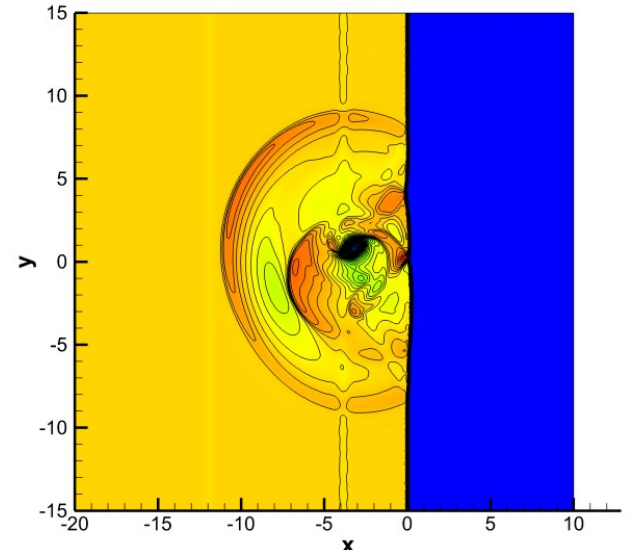

(b) Results of WENOIU3-1MP on randomized grids

Figure 21: Density contours of shock-vortex interaction on two $601 \times 601$ grids by using WENOIU3 schemes (Contours from 0.6 to 2.3 with number 14 ).

The density contours of WENOIU5 schemes are shown in Fig. 22. Because the results of WENOIU5-1MP and -2MP schemes show no qualitative difference on uniform grids, only that of WENOIU5-2MP are displayed. On randomized grids, both schemes accomplish the computations, and the result of WENOIU5-1MP is shown in Fig. 22(b) for representative. From Fig. 22, although the structures on randomized grids resemble to that on uniform grids, tiny oscillations in the former result are observed after the shock. Further investigation in more detail indicates that, the contours by WENOIU5-2MP appear relatively more oscillatory than that of WENOIU5-1MP. Hence, WENOIU5-2MP might 


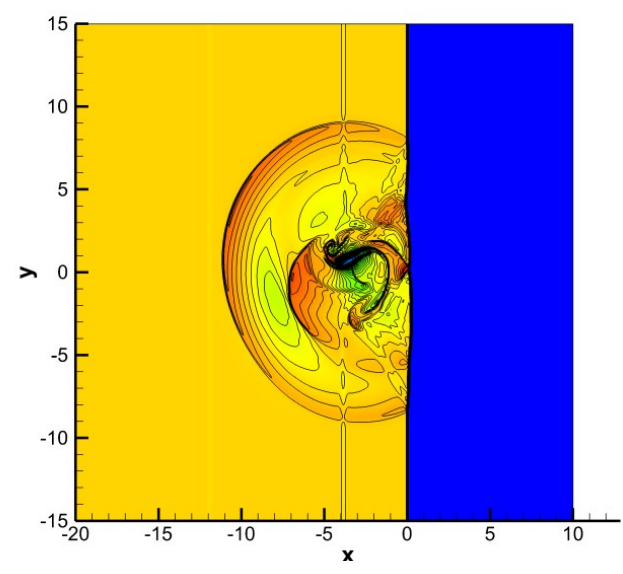

(a) Results of WENOIU5-2MP on uniform grids

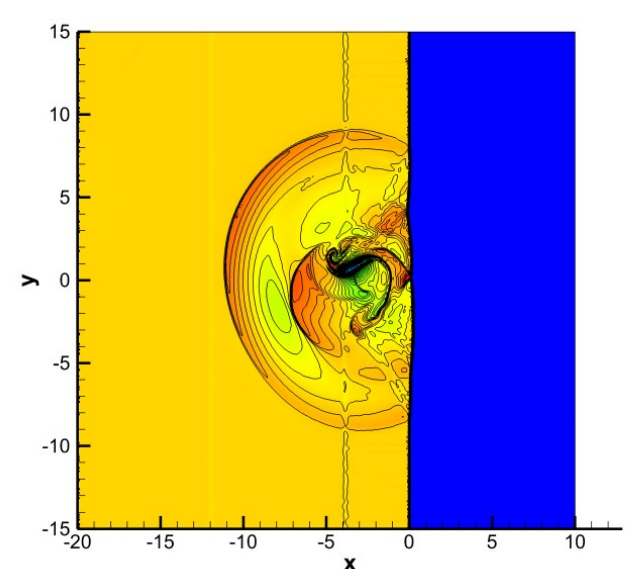

(b) Results of WENOIU5-1MP on randomized grids

Figure 22: Density contours of shock-vortex interaction on two $601 \times 601$ grids by using WENOIU5 schemes (Contours from 0.6 to 2.3 with number 14 ).

be relatively less stable or robust when the grids are distorted.

\section{Concluding remarks and discussions}

With understandings on linear upwind schemes with flux splitting to achieve FSP, new series of nonlinear upwind-biased schemes are developed and abbreviated as WENOIU. In the schemes, the nonlinearity is introduced by the inclusion of midpoints and WENO interpolations. The third- and fifth-order WENOIU schemes are tested by typical examples. The following conclusions are drawn:

(1) The development of WENOIU schemes contributes to methodology to make highorder, upwind nonlinear scheme to achieve FSP. Through the study, the complete third-, fifth- and seventh-order upwind-biased schemes are devised and the first two are investigated in detail. The constructions indicate the successful integration of requirements of FSP and nonlinearity by WENO interpolation under the framework of upwind scheme. Besides, discussions are made regarding proposed schemes, AF-WENO and h-WCNS, in which a general formulation of center scheme with midpoint and nodes included is obtained as a byproduct.

(2) Two of main characteristics of WENOIU schemes, i.e., the accuracy order and achievement of FSP, are validated by numerical tests. Specifically, in order to acquire designed order at first-order critical points, PPM should be casted in nonlinear interpolations, and in addition, the smoothness indicators in WENOIU3 should choose the ones on three-point stencils rather than those on two-point stencils. 
(3) The performances of WENOIU3 and WENOIU5 schemes for applications with shock waves are manifested by three problems. In the problems randomized/locally randomized grids besides uniform/smooth ones are used. On two types of grids, all schemes accomplish the test of supersonic cylinder flow and nearly all of them complete that of Riemann problem and shock-vortex interaction. The only exception is that WENOIU3-2MP fails on randomized grids. On the one hand the results manifest the capability of WENOIU3-1MP and WENOIU5 schemes for solving problems on grids with bad quality, on the other hand the relatively weaker stability of WENOIU3-2MP is also indicated. Generally speaking, WENOIU schemes with two midpoints seems to be less robust or more oscillatory than ones with one midpoint.

One can see from Eqs. (2.13), (2.17), (2.26) and (2.29) that $f_{j+1 / 2}$ takes a special role in proposed schemes. Actually for the computation of $f_{j+1 / 2}$, different flux splitting schemes can be employed, which will not influence the achievement of FSP. Hence the frequentlyused schemes with low dissipation can be employed, e.g., Steger-Warming scheme, Van Leer scheme, AUSM and etc. In the meanwhile, the recent advances in WENO-Z schemes can be applied in interpolations as well. Such issues should deserve attentions in further studies.

It is noticed as well that the tests included in this study are limited. The consideration for test selection is that current study targets to validate the achievement of FSP of proposed nonlinear schemes, and to indicate their capability for solving problems with shock waves and/or vortices on distorted grids. More tests and further explorations are planned in other investigations.

\section{Appendix A}

For completeness, formulas of all possible seventh-order WENOIU schemes are given in a similar way to that in Section 2.3. Likewise, only upwind-biased schemes are concerned.

(1) Scheme with only one midpoint $x_{j+1 / 2}$ included.

The general form of the scheme with one free parameter is

$$
\begin{aligned}
\hat{f}_{j+1 / 2}=\alpha & f_{j+1 / 2}+\left(\frac{5}{1024} \alpha-\frac{1}{140}\right) f_{j-3}+\left(-\frac{21}{512} \alpha+\frac{5}{84}\right) f_{j-2}+\left(\frac{175}{1024} \alpha-\frac{101}{420}\right) f_{j-1} \\
& +\left(-\frac{175}{256} \alpha+\frac{319}{420}\right) f_{j}+\left(-\frac{525}{1024} \alpha+\frac{107}{210}\right) f_{j+1}+\left(\frac{35}{512} \alpha-\frac{19}{210}\right) f_{j+2} \\
& +\left(-\frac{7}{1024} \alpha+\frac{1}{105}\right) f_{j+3} .
\end{aligned}
$$


When $\alpha=1$, the scheme becomes

$$
\hat{f}_{j+1 / 2}=f_{j+1 / 2}+\frac{1}{107520}\left(\begin{array}{c}
-243 f_{j-3}+1990 f_{j-2}-7481 f_{j-1}+8164 f_{j} \\
-341 f_{j+1}-2378 f_{j+2}+289 f_{j+3}
\end{array}\right) .
$$

The corresponding eighth-order $\delta^{c,(1)} f$ is

$$
\delta^{c,(1)} f=\frac{1}{\Delta x}\left(f_{j+1 / 2}-f_{j-1 / 2}\right)+\frac{1}{107520 \Delta x}\left[\begin{array}{c}
-\frac{243}{2}\left(f_{j+4}-f_{j-4}\right)+1261\left(f_{j+3}-f_{j-3}\right) \\
-6069\left(f_{j+2}-f_{j-2}\right)+8841\left(f_{j+1}-f_{j-1}\right)
\end{array}\right] .
$$

(2) Scheme with two midpoints included.

The scheme can be derived as

$$
\hat{f}_{j+1 / 2}=f_{j+1 / 2}+\frac{1}{1260}\left[\begin{array}{l}
\left(-128 f_{j-1 / 2}+404 f_{j+1 / 2}\right) \\
+\left(-2 f_{j-2}+47 f_{j-1}-92 f_{j}-233 f_{j+1}+5 f_{j+2}\right)
\end{array}\right] .
$$

The corresponding eighth-order $\delta^{c,(1)} f$ is

$$
\begin{aligned}
\delta^{c,(1)} f= & \frac{1}{315 \Delta x}\left[-16\left(f_{j+3 / 2}-f_{j-3 / 2}\right)+432\left(f_{j+1 / 2}-f_{j-1 / 2}\right)\right] \\
& \quad+\frac{1}{1260 \Delta x}\left[-\left(f_{j+3}-f_{j-3}\right)+27\left(f_{j+2}-f_{j-2}\right)-189\left(f_{j+1}-f_{j-1}\right)\right] .
\end{aligned}
$$

(3) Scheme with four midpoints included.

The scheme can be derived as

$$
\hat{f}_{j+1 / 2}=f_{j+1 / 2}+\frac{1}{420}\left[\begin{array}{l}
\left(18 f_{j-1}-66 f_{j}-108 f_{j+1}\right) \\
+\left(-3 f_{j-3 / 2}-31 f_{j-1 / 2}+179 f_{j+1 / 2}+11 f_{j+3 / 2}\right)
\end{array}\right] .
$$

The corresponding $\delta^{c,(1)} f$ is

$$
\begin{aligned}
\delta^{c,(1)} f= & \frac{1}{140 \Delta x}\left[3\left(f_{j+2}-f_{i-2}\right)-32\left(f_{j+1}-f_{j-1}\right)\right] \\
& \quad+\frac{1}{840 \Delta x}\left[-3\left(f_{j+5 / 2}-f_{i-5 / 2}\right)-17\left(f_{j+3 / 2}-f_{j-3 / 2}\right)+1218\left(f_{j+1 / 2}-f_{j-1 / 2}\right)\right] .
\end{aligned}
$$

(4) Scheme with six midpoints included.

The scheme can be derived as

$$
\hat{f}_{j+1 / 2}=f_{j+1 / 2}+\frac{1}{26880}\left[3840 f_{j-1}+\left(\begin{array}{l}
-45 f_{j-5 / 2}+501 f_{j-3 / 2}-3874 f_{j-1 / 2} \\
+746 f_{j+1 / 2}-1249 f_{j+3 / 2}+81 f_{j+5 / 2}
\end{array}\right)\right] .
$$

The corresponding $\delta^{c,(1)} f$ is

$$
\delta^{c,(1)} f=\frac{1}{14 \Delta x}\left(f_{j+1}-f_{j-1}\right)+\frac{1}{\Delta x}\left[\begin{array}{c}
-\frac{3}{3584}\left(f_{j+7 / 2}-f_{j-7 / 2}\right)+\frac{209}{17920}\left(f_{j+5 / 2}-f_{j-5 / 2}\right) \\
-\frac{163}{1536}\left(f_{j+3 / 2}-f_{j-3 / 2}\right)+\frac{575}{512}\left(f_{j+1 / 2}-f_{j-1 / 2}\right)
\end{array}\right] .
$$


Table 6: Coefficients $b_{k, l}^{r}$ and $C_{k}^{r}$ in interpolation schemes [32].

\begin{tabular}{||c|c|c|cccc||}
\hline \multirow{2}{*}{$r$} & \multirow{2}{*}{$k$} & \multirow{2}{*}{$C_{k}^{r}$} & \multicolumn{4}{|c||}{$b_{k, l}^{r}$} \\
\cline { 4 - 7 } & & & $l=0$ & $l=1$ & $l=2$ & $l=3$ \\
\hline \multirow{4}{*}{4} & 0 & $1 / 64$ & $-5 / 16$ & $21 / 16$ & $-35 / 16$ & $35 / 16$ \\
\cline { 2 - 7 } & 1 & $21 / 64$ & $1 / 16$ & $-5 / 16$ & $15 / 16$ & $5 / 16$ \\
\cline { 2 - 7 } & 2 & $35 / 64$ & $-1 / 16$ & $9 / 16$ & $9 / 16$ & $-1 / 16$ \\
\cline { 2 - 7 } & 3 & $7 / 64$ & $5 / 16$ & $15 / 16$ & $-5 / 16$ & $1 / 16$ \\
\hline
\end{tabular}

WENO interpolations by Eqs. (2.19)-(2.20) are integrated to evaluate variables on midpoints, where the coefficients in the equations are shown in Table 6.

When order degradation at the first-order critical point is concerned, the following fourth-order PPM can be employed [7] to exert on the nonlinear weights in interpolation

$$
g(\omega)= \begin{cases}C_{k}\left[1-\left(\frac{\omega}{C_{k}}-1\right)^{4}\right], & \omega \leq C_{k}, \\ C_{k}-\left(\frac{1}{C_{k}-1}\right)^{3}\left(\omega-C_{k}\right)^{4}, & \omega>C_{k},\end{cases}
$$

\section{Appendix B}

Consider the center formulation of Eq. (2.37). Given the accuracy order $r=2 n$, the coefficients $a_{l}$ and $b_{l}$ can be either derived by means of Eq. (2.12) or overall center form according to $\left(r, n_{\varepsilon}, n_{0}\right)$ with $r=n_{\varepsilon}+n_{0}-1$, and the consequences are summarized in Table 7.

As mentioned in Section 2.4, the formulation by Eq. (2.37) at $(r=2 n, 2 n, 1)$ and with $\alpha=1$ will result in AF-WENO [43], and it will yield form with less grid points possibly such as H-WCNS $[18,44]$ and even WCNS [17] by specific choice of $\alpha$. The latter results are summarized in Table 8, where the superscript of $\alpha$ indicates the reference that has

Table 7: Coefficients $a_{l}$ and $b_{l}$ according to $\left(r, n_{\varepsilon}, n_{0}\right)$ in Eq. (2.37).

\begin{tabular}{||c|c|c|c|c|c|c|c||}
\hline$\left(r, n_{\varepsilon}, n_{0}\right)$ & $a_{l}$ & $a_{2}$ & $a_{3}$ & $a_{4}$ & $b_{l}$ & $b_{2}$ & $b_{3}$ \\
\hline$(4,4,1)$ & $-\frac{9}{16} \alpha+\frac{7}{12}$ & $\frac{1}{16} \alpha-\frac{1}{12}$ & - & - & - & - & - \\
\hline$(4,2,3)$ & $-\frac{2}{3} \alpha+\frac{13}{18}$ & - & - & - & $\frac{1}{6} \alpha-\frac{2}{9}$ & - & - \\
\hline$(6,6,1)$ & $-\frac{75}{128} \alpha+\frac{37}{60}$ & $\frac{25}{256} \alpha-\frac{2}{15}$ & $-\frac{3}{256} \alpha+\frac{1}{60}$ & - & - & - & - \\
\hline$(6,4,3)$ & $-\frac{3}{4} \alpha+\frac{17}{20}$ & $-\frac{1}{20} \alpha+\frac{23}{300}$ & - & - & $\frac{3}{10} \alpha-\frac{32}{75}$ & - & - \\
\hline$(6,2,5)$ & $-\frac{32}{45} \alpha+\frac{1067}{1350}$ & - & - & - & $\frac{2}{9} \alpha-\frac{83}{270}$ & $-\frac{1}{90} \alpha+\frac{23}{1350}$ & - \\
\hline$(8,8,1)$ & $-\frac{1225}{2048} \alpha+\frac{533}{840}$ & $\frac{245}{2048} \alpha-\frac{139}{840}$ & $-\frac{49}{2048} \alpha-\frac{29}{840}$ & $\frac{5}{2048} \alpha-\frac{1}{280}$ & - & - & - \\
\hline$(8,6,3)$ & $-\frac{25}{32} \alpha+\frac{379}{420}$ & $-\frac{5}{64} \alpha+\frac{13}{105}$ & $\frac{1}{448} \alpha-\frac{11}{2940}$ & - & $\frac{5}{14} \alpha-\frac{128}{245}$ & - & - \\
\hline$(8,4,5)$ & $-\frac{4}{5} \alpha-\frac{1961}{2100}$ & $-\frac{4}{35} \alpha-\frac{2711}{14700}$ & - & - & $\frac{2}{5} \alpha-\frac{104}{175}$ & $\frac{1}{70} \alpha-\frac{88}{3675}$ & - \\
\hline$(8,2,7)$ & $-\frac{128}{175} \alpha+\frac{30251}{36750}$ & - & - & - & $\frac{1}{4} \alpha-\frac{789}{2240}$ & $-\frac{1}{50} \alpha+\frac{659}{21000}$ & $\frac{1}{700} \alpha-\frac{2711}{1176000}$ \\
\hline
\end{tabular}


Table 8: Dispersion relation of Eq. (2.37) at $\left(r, n_{\varepsilon}, n_{0}\right)$ and $\alpha$ for less grids formulation.

\begin{tabular}{|c|c|c|c|c|}
\hline$\left(r, n_{\varepsilon}, n_{0}\right)$ & \multicolumn{3}{|l|}{ Dispersion relation } & $\alpha$ for less grids \\
\hline$(4,4,1)$ & \multicolumn{3}{|l|}{$\left(-\frac{5}{4} \alpha+\frac{4}{3}\right) \sin (\kappa)+\left(\frac{1}{8} \alpha-\frac{1}{6}\right) \sin (2 \kappa)+2 \alpha \sin \left(\frac{1}{2} \kappa\right)$} & $\frac{4}{3}$ \\
\hline$(4,2,3)$ & $\left(\frac{4}{3} \alpha-\frac{16}{9}\right) \cos \left(\frac{1}{2} \kappa\right)^{2}+\left(-\frac{8}{3} \alpha+\frac{26}{9}\right) \cos \left(\frac{1}{2} \kappa\right)+\frac{4}{3} \alpha+\frac{8}{9}$ & \multicolumn{2}{|c|}{$\frac{8}{9} \sin \left(\frac{1}{2} \kappa\right)$} & $\frac{4}{3}^{[18]}, \frac{13}{12}^{[17]}$ \\
\hline$(6,6,1)$ & \multicolumn{3}{|c|}{$\left(-\frac{175}{128} \alpha+\frac{3}{2}\right) \sin (\kappa)+\left(\frac{7}{32} \alpha-\frac{3}{10}\right) \sin (2 \kappa)+\left(-\frac{3}{128} \alpha+\frac{1}{30}\right) \sin (3 \kappa)+2 \alpha \sin \left(\frac{1}{2} \kappa\right)$} & $\frac{64}{45}{ }^{[18]}$ \\
\hline$(6,4,3)$ & $\left.\begin{array}{l}\left(-\frac{4}{5} \alpha+\frac{92}{75}\right) \cos \left(\frac{1}{2} \kappa\right)^{3}+\left(\frac{12}{5} \alpha-\frac{256}{75}\right) \cos \left(\frac{1}{2} \kappa\right)^{2} \\
+\left(-\frac{12}{5} \alpha+\frac{62}{25}\right) \cos \left(\frac{1}{2} \kappa\right)+\frac{4}{5} \alpha+\frac{128}{75}\end{array}\right]$ & \multicolumn{2}{|c|}{$\sin \left(\frac{1}{2} \kappa\right)$} & $\frac{64}{45}^{[18]}, \frac{23}{15}{ }^{[44]}$ \\
\hline$(6,2,5)$ & $\begin{array}{l}\left(-\frac{16}{45} \alpha+\frac{368}{675}\right) \cos \left(\frac{1}{2} \kappa\right)^{4}+\left(\frac{32}{15} \alpha-\frac{676}{225}\right) \cos \left(\frac{1}{2} \kappa\right)^{2} \\
+\left(-\frac{128}{45} \alpha+\frac{2134}{675}\right) \cos \left(\frac{1}{2} \kappa\right)+\frac{16}{15} \alpha+\frac{292}{225}\end{array}$ & \multicolumn{2}{|c|}{$\sin \left(\frac{1}{2} \kappa\right)$} & $\frac{23}{15}^{[44]}, \frac{1067}{960}^{[17]}$ \\
\hline$(8,8,1)$ & \multicolumn{3}{|c|}{$\begin{array}{l}\left(-\frac{735}{512} \alpha+\frac{8}{5}\right) \sin (\kappa)+\left(\frac{147}{512} \alpha-\frac{2}{5}\right) \sin (2 \kappa)+\left(-\frac{27}{512} \alpha+\frac{8}{105}\right) \sin (3 \kappa) \\
+\left(\frac{5}{1024} \alpha-\frac{1}{140}\right) \sin (4 \kappa)+2 \alpha \sin \left(\frac{1}{2} \kappa\right)\end{array}$} & $\frac{256}{175}$ \\
\hline$(8,6,3)$ & \multicolumn{3}{|c|}{$\begin{array}{l}\left(-\frac{45}{32} \alpha+\frac{109}{70}\right) \sin (\kappa)+\left(-\frac{9}{56} \alpha+\frac{25}{98}\right) \sin (2 \kappa)+\left(\frac{1}{224} \alpha-\frac{11}{1470}\right) \sin (3 \kappa) \\
+\left(\frac{9}{7} \alpha+\frac{256}{245}\right) \sin \left(\frac{1}{2} \kappa\right)+\left(\frac{5}{7} \alpha-\frac{256}{245}\right) \sin \left(\frac{3}{2} \kappa\right)\end{array}$} & $\frac{256}{175}, \frac{176}{105}^{[44]}$ \\
\hline$(8,4,5)$ & \multicolumn{2}{|c|}{$\begin{array}{l}\left(\frac{16}{35} \alpha-\frac{2816}{3675}\right) \cos \left(\frac{1}{2} \kappa\right)^{4}+\left(-\frac{64}{35} \alpha+\frac{10844}{3675}\right) \cos \left(\frac{1}{2} \kappa\right)^{3} \\
+\left(\frac{96}{35} \alpha-\frac{14656}{3675}\right) \cos \left(\frac{1}{2} \kappa\right)^{2}+\left(-\frac{64}{35} \alpha+\frac{5594}{3675}\right) \cos \left(\frac{1}{2} \kappa\right)+\frac{16}{35} \alpha+\frac{8384}{3675}\end{array}$} & $\sin \left(\frac{1}{2} \kappa\right)$ & $\frac{176}{105}^{[44]}, \frac{2711}{1680}$ \\
\hline$(8,2,7)$ & \multicolumn{2}{|c|}{$\begin{array}{l}\left(\frac{32}{175} \alpha-\frac{5422}{18375}\right) \cos \left(\frac{1}{2} \kappa\right)^{6}+\left(-\frac{32}{35} \alpha+\frac{5317}{3675}\right) \cos \left(\frac{1}{2} \kappa\right)^{4} \\
+\left(\frac{96}{35} \alpha-\frac{14656}{3675}\right) \cos \left(\frac{1}{2} \kappa\right)^{2}+\left(-\frac{512}{175} \alpha+\frac{60502}{18375}\right) \cos \left(\frac{1}{2} \kappa\right)+\frac{32}{35} \alpha+\frac{1891}{1225}\end{array}$} & $\sin \left(\frac{1}{2} \kappa\right)$ & $\frac{2711}{1680}, \frac{30251}{26880}[44]$ \\
\hline
\end{tabular}

proposed the same scheme. In case of $r=8$, the choice of $\alpha$, which makes the resulted scheme have the 10th-order accuracy is not included.

To facilitate potential optimization attempt on dispersion relations of Eq. (2.37), the analytic expressions of relations are derived and shown in Table 8 as well.

\section{Acknowledgements}

This study is sponsored by the project of National Numerical Wind-tunnel of China under the grant number No. NNW2019ZT4-B12. The second author thanks for the support of National Natural Science Foundation of China under the Grant No. 11802324. The corresponding author thanks for the contribution of Dr. Qilong Guo on the incipient 1-D computations, and he is also grateful to Prof. Kun $\mathrm{Xu}$ for his efforts on the revision of the manuscript as well as Dr. Pengxin Liu for supplementary computations.

\section{References}

[1] X.-D. LIU, S. Osher, AND T. CHAN, Weighted essentially non-oscillatory schemes, J. Comput. Phys., 115 (1994), pp. 200-212.

[2] G. S. JIANG, AND C.-W. SHU, Efficient implementation of weighted ENO schemes, J. Comput. Phys., 126 (1996), pp. 202-228.

[3] D. S. BALSARA AND C.-W. SHU, Monotonicity preserving weighted essentially non-oscillatory schemes with increasingly high order of accuracy, J. Comput. Phys., 160 (2000), pp. 405-452.

[4] X. ZHANG ANND C.-W. SHU, Positivity-preserving high order finite difference WENO schemes for compressible Euler euqations, J. Comput. Phys., 231 (2012), pp. 2245-2258. 
[5] A. K. Henrick, T. D. Aslam, AND J. M. Powers, Mapped weigted essentially non-oscillatory schemes: achieving optimal order near critical points, J. Comput. Phys., 207 (2005), pp. 542-567.

[6] H. FENG, F. HU AND R. WANG, A new mapped weighted essentially non-oscillatory scheme, J. Sci. Comput., 51 (2012), pp. 449-473.

[7] Q. LI, P. LIU, AND H. ZHANG, Piecewise polynomial mapping method and corresponding WENO scheme with improved resolution, Commun. Comput. Phys., 18 (2015), pp. 1417-1444.

[8] R. Borges, M. CARMONA, B. COsTA, AND W. S. DON, An improved weighted essentially non-oscillatory scheme for hyperbolic conservation laws, J. Comput. Phys., 227 (2008), pp. 31913211.

[9] M. CAstro, B. CostA, AND W. S. DON, High order weighted essentially non-oscillatory WENO-Z schemes for hyperbolic conservation laws, J. Comput. Phys., 230 (2011), pp. 17661792.

[10] F. ACKer, R. Borges, And B. Costa, An improved WENO-Z scheme, J. Comput. Phys., 313 (2016), pp. 726-753.

[11] E. M. TAYLOR, M. WU, AND M. P. MARTín, Optimization of nonlinear error for weighted essentially non-oscillatory methods in direct numerical simulations of compressible turbulence, J. Comput. Phys., 223 (2006), pp. 384-397.

[12] Z.-S. SUN, Y.-X. REN, C. LARRICQ, S.-Y. ZHANG, AND Y.-C. YANG, A class of finite schemes with low dispersion and controllable dissipation for DNS of compressible turbulence, J. Comput. Phys., 230 (2011), pp. 4616-4635.

[13] Q. Li, Q. GUO, D. SUN, P. LIU, AND H. ZHANG, A fourth-order symmetric WENO scheme with improved performance by new linear and nonlinear optimizations, J. Sci. Comput., 71 (2017), pp. 109-143.

[14] S. K. LELE, Compact finite difference schemes with spectral-like resolution, J. Comput Phys., 103 (1992), pp. 16-42.

[15] M. R. VisBal AND D. V. GAITONDE, On the use of high-order finite-difference schemes on curvilinear and deforming meshes, J. Comput. Phys., 181 (2002), pp. 155-195.

[16] T. Nonomura, N. LizUKA, AND K. FUjII, Freestream and vortex preservation properties of high-order WENO and WCNS on curvilinear grids, Comput. Fluids, 39 (2010), pp. 197-214.

[17] X. DenG, AND H. ZHANG, Developing high-order weighted compact nonlinear schemes, J. Comput. Phys., 165 (2000), pp. 22-44.

[18] X. DenG, Y. JiAnG, M. MAO, H, LiU, S. Li, AND G. TU, A family of hybrid cell-edge and cell-node dissipative compact schemes satisfying geometric conservation law, Comput. Fluids, 116 (2015), pp. 29-45.

[19] M. VINOKUR, Conservative equations of gasdynamics in curvilinear coordinate systems, J. Comput. Phys., 14 (1974), pp. 105-125.

[20] T. H. PUlliam AND J. L. STEGER, On implicit finite-difference simulations of three dimensional flow, AIAA78-10, (1978).

[21] P. D. THOMAS AND C. K. LOMBARD, The geometric conservation law-a link between finitedifference and finite-volume methods of flow computation on moving grids, AIAA 78-1208, (1978).

[22] P. D. THOMAS AND K. L. NEIER, Navier-Stokes simulation of three-dimensional hypersonic equilibrium flows with ablation, J. Spacecraft Rocket, 27 (1990), pp. 143-149.

[23] M. VINOKUR AND H. C. YEE, Extension of efficient low dissipation high order schemes for $-3 D$ curvilinear moving grids, NASA-TM-209598, (2000).

[24] X. DenG, M. MAO, G. TU, H. LIU, AND H. ZHANG, Geometric conservation law and applications to high-order finite difference schemes with stationary grids, J. Comput. Phys., 230 (2011), pp. 1100-1115. 
[25] T. Nonomura, D. TeraKado, Y. ABE, AND K. FujII, A new technique for freestream preservation of finite-difference WENO on curvilinear grid, Comput. Fluids, 107 (2015), pp. 242-255.

[26] Q. LI, D. SUN, AND P. LIU, Further study on errors in metric evaluation by linear upwind schemes with flux splitting in stationary grids, Commun. Comput. Phys., 22 (2017), pp. 64-94.

[27] H. C. YEE, Upwind and symmetric shock-capturing schemes, NASA TM-89464, (1987).

[28] T. KaWAMURA, H. TAKAMI, AND K. KUWAHARA, New higher order upwind scheme for incompressible Navier-Stokes equations, Fluid Dyn. Res., 1 (1985), pp. 145-162

[29] X. DENG, H. MAEKAWA, AND C. SHEN, A class of high-order dissipative compact scheme, AIAA 96-1972, 1996.

[30] S.-T. YU, L. S. Hultgren, AND N.-S. LIU, Direct calculations of waves in fluid flows using high-order compact difference scheme, AIAA J., 32 (1994), pp. 1766-1773.

[31] A. K. DE, AND V. ESWARAN, Analysis of a new high resolution upwind compact scheme, J. Comput. Phys., 218 (2006), pp. 398-416.

[32] C.-W. SHU, High order weighted essentially nonoscillatory schemes for convection dominated problems, SIAM Rev., 51 (2009), pp. 82-126

[33] X. ZHONG, High-order finite-difference schemes for numerical simulation of hypersonic boundarylayer transition, J. Comput. Phys., 144 (1998), pp. 662-709.

[34] C.-W. SHU AND S. OSHER, Efficient implementation of essentially non-oscillatory shock capturing schemes, J. Comput. Phys., 77 (1988), pp. 439-471.

[35] Y. ABE, N. LizUKA, T. NONMURA, AND K. FUjII, Symmetric-conservative metric evaluations for high-order finite difference scheme with the GCL identities on three-dimensional moving and deforming mesh, Proceedings of Seventh International Conference on Computational fluid Dynamics, ICCFD7-2801, 2012.

[36] M.-L. WONG, AND S. K. LELE, High-order localized dissipation weighted nonlinear scheme for shock- and interface-capturing in compressible flows, J. Comput. Phys., 339 (2017), pp. 179-209.

[37] S. ZHANG, S. JIANG AND C.-W. SHU, Development of nonlinear weighted compact schemes with increasingly higher order accuracy, J. Comput. Phys., 227 (2008), pp. 7294-7321.

[38] C. K. W. TAM, AND J. C. WEBB, Dispersion-relation-preserving finite difference schemes for computational acoustics, J. Comput. Phys., 107 (1993), pp. 262-281.

[39] Z. YAN ETC., Development of 3rd-order HWCNS and its application in hypersonic flow, Acta Aeronautica et Astronautica Sinica, 36 (2015), pp. 1460-1470.

[40] S. Pirozzoli, On the spectral properties of shock-capturing schemes, J. Comput. Phys., 219 (2006), pp. 489-497.

[41] A. N. LyUbinOV, AND V. V. RusAnOV, Gas flow past blunt bodies, NASA TT-F715, (1973).

[42] Y. JIANG, C. -W. SHU, AND M.-P. ZHANG, An alternative formulation of finite difference weighted ENO schemes with Lax-Wendorff time discretization for conservative laws, J. Sci. Comput., 35 (2013), pp. 1137-1160.

[43] Y. JIANG, C. -W. SHU, AND M.-P. ZHANG, Free-stream preserving finite difference schemes on curvilinear meshes, Methods Appl. Anal., 21 (2014), pp. 1-30.

[44] T. NONOMURA AND K. FUJII, Robust explicit formulation of weighted compact nonlinear scheme, Comput. Fluids, 85 (2013), pp. 8-18. 